\title{
The distribution of Pteridophytes (ferns and lycophytes) in Sicily
}

Filippo Rizzo

Via Libertà 63, 90018 Termini Imerese, Palermo, Italy; e-mail: filippo.rizzo14@outlook.it

\begin{abstract}
In this study, an updated list of Sicilian ferns and lycophytes is presented, with ecological information and an in-depth analysis on provincial distribution. The Pteridophytes present in Sicily are 58 (including species and subspecies), 6 Lycopodiopsida and 52 Polypodiopsida, including 4 allochthonous naturalized taxa (Azolla cristata, Azolla filiculoides, Cyrtomium falcatum and Nephrolepis cordifolia). There are also 4 further uncertain taxa (Asplenium trichomanes subsp. pachyrachis, Dryopteris borreri, Dryopteris cambrensis subsp. insubrica and Polypodium vulgare). The largest family is that of Aspleniaceae, which contains the larger genus Asplenium. With regard to the distribution, the province of Palermo has the largest number of taxa, followed by the provinces of Messina, Catania and Trapani. Overall, the ferns are unevenly distributed, with the Northern provinces having a higher number of taxa.
\end{abstract}

KEY WORDS Ferns; Pteridophyta; distribution; Sicily; Italy.

Received 15.09.2020; accepted 22.12.2020; published online 15.02.2021

\section{INTRODUCTION}

According to the most recent taxonomic studies (Pteridophyte Phylogeny Group, 2016; Marchetti, 2017), Pteridophyta is a paraphyletic taxon, consisting of two classes: "Lycopodiopsida" (which include Lycopodiaceae, Selaginellaceae e Isoetaceae) and "Polypodiopsida" (which include ferns in a broad sense and related species such as Equisetaceae, Ophioglossaceae and other families not present in Italy).

The indigenous and naturalized Pteridophytes in Europe (including subspecies) exceed 180 units by a little. Italy has about 130 pteridophytes, so it looks very rich, sharing the record with France and Spain (Marchetti, 2003). Since the last specific update on pteridological flora (Troia et al., 2011), Sicily has 55 pteridophytes species and subspecies.
The purpose of this work is to update and deepen the state of knowledge on Pteridophytes present in Sicily and to define their distribution in greater detail, integrating historical data with the most recent reports.

\section{MATERIAL AND METHODS}

The species information sheets of Sicilian ferns, updated and produced in this study (Annex 1), was drawn up on the basis of the most recent floras, from which the descriptive information was also taken: Giardina et al. (2007), Raimondo \& Spadaro (2009), Raimondo et al. (2010), Troia et al. (2011), Marchetti (2015), and Pignatti et al. (2017-19). For each species and subspecies a descriptive sheet has been created with various information (Fig. 1). The referenced nomenclature, as well as the biological 
form and chorological type, are those used in Pignatti et al. (2017-19) and subsequent updates. Within the families, the genera, the species and the subspecies (except the nominal subspecies which are placed first) are arranged in alphabetical order. The regional frequency indices are as follows: $\mathrm{RR}$ $=$ very rare $\mathrm{R}=$ rare $\mathrm{C}=$ common $; \mathrm{CC}=$ very common. The regional IUCN categories attributed to each taxon (except alien species) were used to assess the state of conservation (Raimondo et al., 2011; García et al., 2017). The acronyms of the Italian regions are those used by Conti et al. (2005), instead for the Sicilian provinces the official acronyms were used: PA (prov. Palermo); TP (prov. Trapani); ME (prov. Messina); CT (prov. Catania); EN (prov. Enna); AG (prov. Agrigento); CL (prov. Caltanissetta); RG (prov. Ragusa); SR (prov. Siracusa). For each taxon the local reports made in the Sicilian territory have been included, divided by province, except for widely distributed species whose presence is ascertained throughout the region. On the map, the Sicilian provinces where the autochthonous taxa are present are indicated in green. The yellow color indicates the taxa whose presence is uncertain, not recently confirmed and / or the actual distribution is not yet defined and for which it is necessary to make further investigations to determine its presence in the territory or alternatively the extinction. Uncertain individual locations are marked with a question mark (?). The blue indicates the allochthonous taxa. Hybrids are not taken into consideration.

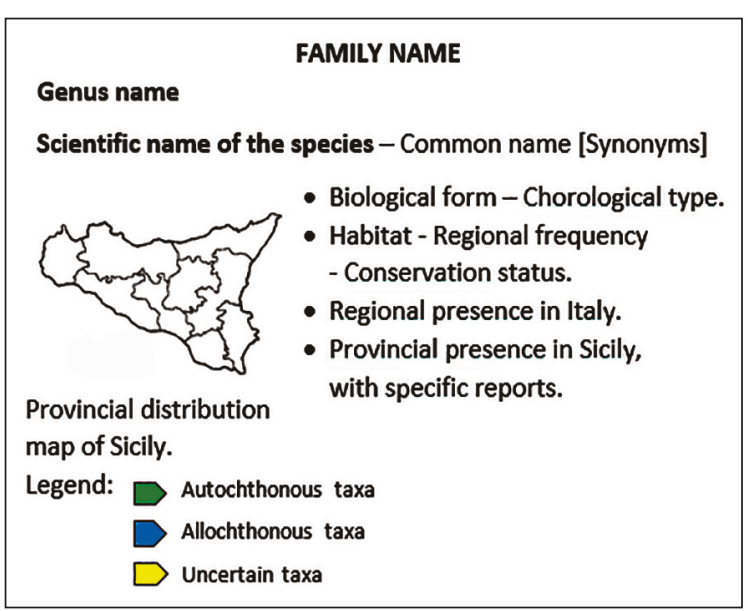

Figure 1. Species type information sheet for the description of the species and subspecies.
The local reports have been extrapolated from various floristic and vegetational studies, as well as from specialized online forums such as Acta Plantarum (www.actaplantarum.org), Wikiplantbase \#Sicilia (http://bot.biologia.unipi.it/wpb/sicilia/in dex.html) and the Facebook group of Flora Spontanea Siciliana (www.facebook. com/groups/ floraspontaneasiciliana). These bibliographic sources are indicated at the end of the sheets. The data derived from herbarium samples are indicated with the herbarium's acronym (according to the Index Herbariorum) and an exclamation point, placed immediately after the locality to which they refer, adding the name of the collector if available (e.g., Lojacono PAL!). Personal field observations are marked with an exclamation mark (!).

Further additional information is given as notes. As for the Lycopodiaceae in Sicily, Lycopodiella cernua (L.) Pic. Serm. was reported in the Syracuse area, at Palazzo Acreide (Fiori, 1943; Giardina et al., 2007). The report was based on only one sterile specimen collected in 1904 in a bush (Pignatti et al., 2017-19), no longer found, even in recent research (Troia \& Greuter, 2015a) and was considered a random presence. For this reason, this species and family have to be excluded in Sicily.

\section{RESULTS AND DISCUSSION}

The Sicilian pteridophytes are made up of 2 classes, 16 families, 26 genera and 58 taxa (species and subspecies). The Lycopodiopsida class are represented by 2 families (Isoëtaceae and Selaginellaceae), with 2 genera and a total of 6 taxa; the Polypodiopsida class is represented by 14 families, 24 genera and 52 taxa. In the latter class there are included 4 alien naturalized taxa (Azolla cristata, Azolla filiculoides, Cyrtomium falcatum and Nephrolepis cordifolia). In Sicily there are also 4 further uncertain taxa (Asplenium trichomanes subsp. pachyrachis, Dryopteris borreri, Dryopteris cambrensis subsp. insubrica and Polypodium vulgare). The uncertain taxa are not included in the number of taxa occurring in Sicily and have not been used to prepare Figs. 2-5.

As reported in the graphs (Figs. 2, 3) the most numerous family is that of the Aspleniaceae, with 16 taxa, which also contains the most numerous genus Asplenium (11 taxa), in second place the 


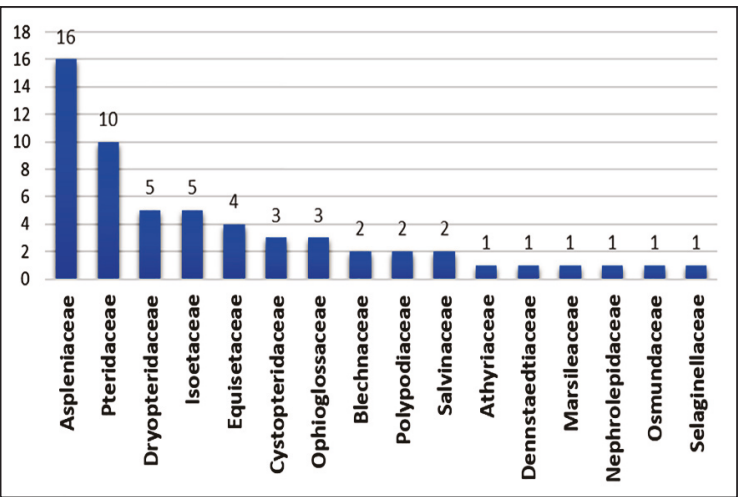

Figure 2. Number of taxa (species and subspecies) of pteridophytes in Sicily, by family.

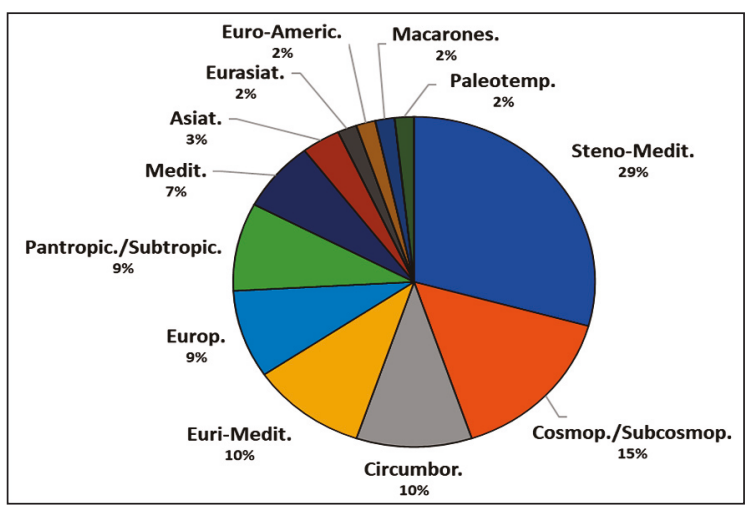

Figure 4. Chorological graph of the Sicilian pteridophytes.

Pteridaceae family, with 10 taxa. From the chorological point of view (Fig. 4) about half of the taxa are widely distributed and the other half has a Mediterranean distribution. These results comply with those reported by Troia et al. (2011).

Regarding the distribution of pteridophytes in Sicily, the province of Palermo, with 47 taxa (Fig. $5)$, is the one with the highest concentration of pteridophytes, followed by the provinces of Messina and Catania, with respectively 43 and 38 taxa. The province of Trapani also has numerous species, 35 taxa, largely due to the high floristic biodiversity of the Egadi Islands and especially the Island of Pantelleria (e.g., Cheilanthes guanchica, Asplenium marinum, Asplenium balearicum). There are numerous species widely distributed throughout the Sicilian territory (e.g., Selaginella denticulata, Equisetum ramosissimum, Adiantum capillus-veneris, Pteridium aquil-

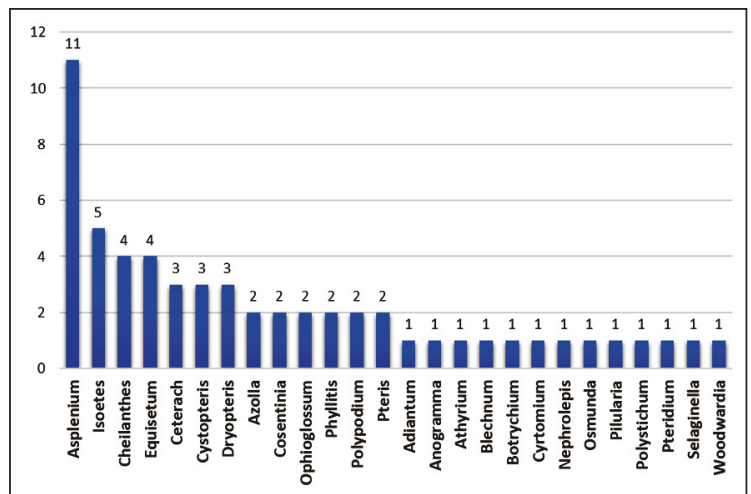

Figure 3. Number of taxa (species and subspecies) of pteridophytes in Sicily, by genus.

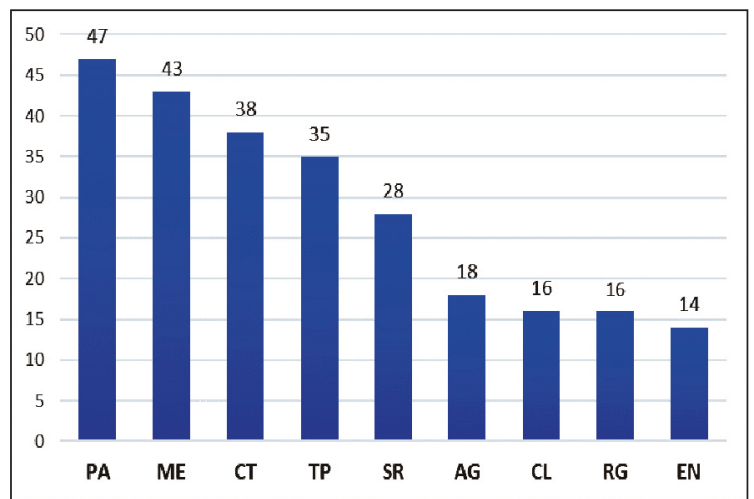

Figure 5. Number of taxa of pteridophytes occurring in the Sicilian provincies, in decreasing order

inum, Asplenium onopteris, etc.), but also other ones with limited or point-like areas (e.g., Isoëtes todaroana, Chelianthes guanchica, Asplenium marinum, etc.).

In general it can be noted that there is a clear separation, in the number of taxa present, between the rich provinces of northern Sicily (PA, ME, CT and TP) compared to the poor ones of southern Sicily (SR, AG, CL, RG e EN). Moreover, most of the taxa of Central-Southern Sicily are widely distributed and present throughout the territory. This trend could be due to the lack of adequate environmental (geographic and climatic) conditions in the south to host more demanding species which remain relegated to the northern part of Sicily. Another factor to take into consideration is the lack of data for some provinces, which are poorly studied and which require further cognitive insights from a floristic point of view. 


\section{Annex 1: Species information sheets}

ISOËTACEAE Dumort

Genus Isoëtes L.

\section{Isoëtes duriei Bory}

Isoëtes durieui Bory; Isoetella durieui (Bory) Gennari; Calamaria durieui (Bory) Kuntze - Calamaria di Durieu

G bulb - Steno-Medit.-Occid.

Wet meadows, seasonally saturated with water or flooded, ponds, wet rocky places, from 0 to 900 m a.s.1. - R - EN.

LIG, TOS, LAZ, MOL?, CAM, CAL, SAR, SIC.

PA: M. Cuccio al Piano della Montagna, Ficuzza al Gorgo del Drago presso Godrano (Troia PAL!), Ficuzza al bivio Lupo (Troia PAL!), Ficuzza al Fiume San Leonardo, Ficuzza a NW del rifugio Val di Conti, Dingoli presso Piana degli Albanesi, Schillizzi nei pressi di Piana degli Albanesi (Troia \& Napolitano PAL!), Piana degli Albanesi a Dingoli presso casa Sala, Bosco e margi di Tumminia, Bolognetta C.da Gasaca (Lordica) (Troia PAL!), Bosco di Gibilmanna, Madonie C.da Pianetti (presso Gibilmanna) (Raimondo PAL!), Sovachella pressa Petralia Soprana, Madonie a Portella Colla sopra portella Mandarini (Troia PAL!), Castelbuono (PAL!); TP: Pantelleria alle fumarole "Le Favare" (Ross PAL!; G. Moggi \& E. Nardi FI!), Pantelleria presso la cima di M. Grande (Sommier FI!), Pantelleria alle Balate (Sommier FI!), Pantelleria M. Gibele (Sommier FI!), Pantelleria nel letto del torrente di Rakhab (Sommier FI!), Alcamo a Passarello (PAL!), Bosco di Scorace (Troia PAL!), Trapanese; ME: Lipari vetta di M. S. Angelo presso

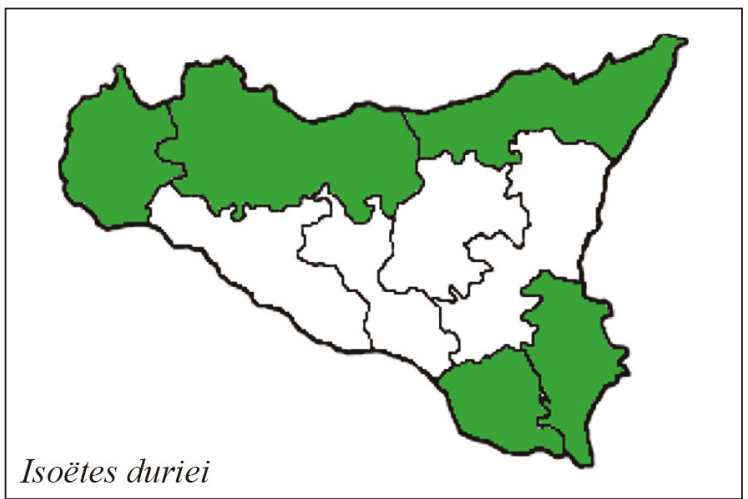

le fosse della Neve (Lojacono PAL!), San Corrado, Sotto Campo Inglese, Salice, Puntale CaraccioloZafferia, Serra Pedra-Larderia, Castanea al Piano di Ravelli, Scoppo, Casazza, Camaro, nei pressi di Messina (Ross FI!); RG: ragusano; SR: M. Lauro, Francofonte: Laghetto C.da Passanatello, Bosco Pisano (Giardina PAL!) (Lojacono, 1909; Ferro et al., 1992; Raimondo et al., 1992; Oddo \& Bellini, 1994; Gianguzzi, 1999, 2003; Picone et al., 2003; Troia, 2005; Giardina et al., 2007, Troia et al., 2012a; Troia \& Greuter, 2015b; Domina et al., 2019, Cambria \& Tavilla, 2020).

\section{Isoëtes histrix Bory}

Cephaloceraton histrix (Bory) Gennari - Calamaria istrice

G bulb - Steno-Medit.-Atlant.

Wet meadows, seasonally saturated with water or flooded, ponds, from 0 to 1000 m s.l.m - R - EN.

TOS, LAZ, CAM, PUG, CAL, SAR, SIC.

PA: Ficuzza al Lupo, C.da Strasatto tra Giacalone e Poggio S. Francesco, Cefalù a Finale (PAL!), Mazzaforno (Cefalù), Mongiarrati (Isnello), Madonie, Bosco della Tumminia, Ogliastro, S. Guglielmo presso Castelbuono, Finale (Pollina), Piana della Stoppa sopra Misilmeri; TP: Alcamo alla Fico (PAL!), Alcamo al Passarello (Citarda PAL!), tra Alcamo e Partinico (Todaro FI!), Bosco di Calatafimi (Citarda PAL!), Bosco Angimbè, Isola Grande dello Stagnone in C.da Carco; ME: Castanea a Ravelli (Caruel FI!), Salice (Peloritani); SR: Cozzo Ogliastro presso Sortino, Francofonte: Laghetto C.da Passanatello, Bosco Pisano (Giardina PAL!), Buccheri nei pressi di Zelkova sicula (Troia PAL!) (Lojacono, 1909; Ottonello \& Catanzaro, 1985; Raimondo et al., 1992; Picone et al., 2003; Marchetti, 2004; Troia,

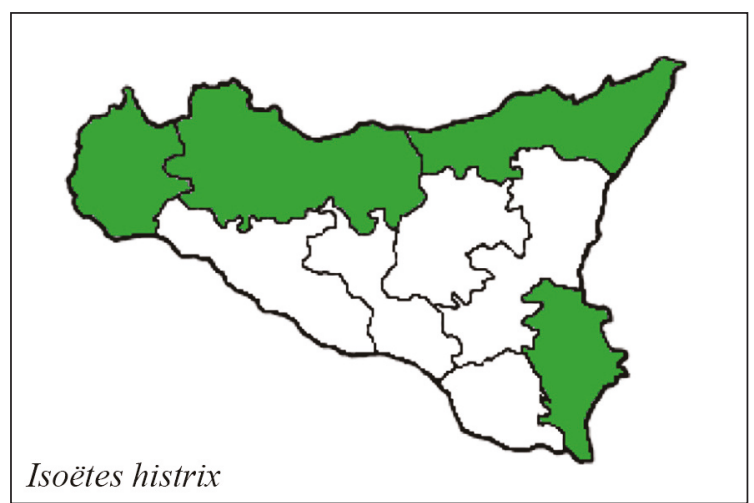


2005; Giardina et al., 2007; Troia et al., 2012a; Troia $\&$ Greuter, 2015b; Domina et al., 2019).

Isoëtes subinermis (Durieu) Cesca \& Peruzzi Cephaloceraton hystrix var. subinerme Gennari; $C$. gymnocarpum Gennari; Isoëtes gymnocarpa (Gennari) A. Braun; I. hystrix subsp. subinermis Dur.; I. histrix var. brevispina A. Braun; I. sicula Tod.; I. histrix subsp. sicula (Tod.) P. Fourn. - Calamaria con frutto nudo

G bulb - S-Medit.

Wet meadows, seasonally saturated with water or flooded, ponds, from 0 to $1000 \mathrm{~m}$ a.s.l. - R - EN.

TOS?, LAZ?, PUG?, CAL, SIC.

PA: Ficuzza al Lupo (Lojacono PAL!), Bosco della Tumminia fra Ogliastro e Ventimiglia (PAL!), Madonie (Citarda PAL!), Bosco Mirto presso Partinico, Bolognetta, vicino la SS 121 al Km 234+200, Cefalù al Finale (PAL!), Contrada Pianetti, Gibilmanna, Madonie (Raimondo PAL!); TP: Alcamo a Passarello (PAL!), Bosco di Calatafimi (PAL!), Bosco di Scorace presso Buseto Palizzolo (Troia PAL!), tra Roccolino sottano e Torre Grimesi, Roccolino, Pantani di Anguillara (Calatafimi, Segesta) (Troia \& Marrone PAL!), Roccolino; CT/CL: Bivio Cimia presso S. Cono (Troia et al. PAL!); SR: Bucchieri, M. Lauro; (Troia, 2005; Troia et al., 2012a; Troia \& Greuter, 2015b; Troia et al., 2016; Troia et al., 2019).

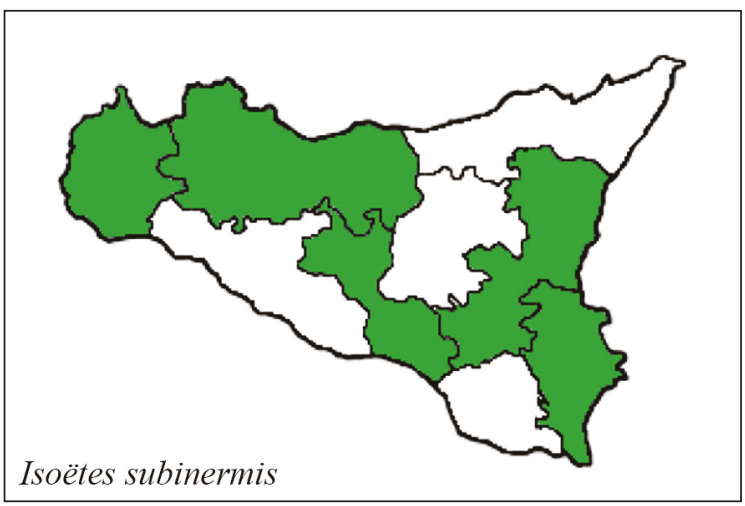

Isoëtes todaroana Troìa \& Raimondo

Isoëtes iapygia Ernandes, Beccarisi \& Zuccarello Calamaria di Todaro

G bulb - SO-Steno-Medit. (Italia and Grecia).

Wet meadows, seasonally saturated with water or flooded and moist limestone rocky places, from 0 to $150 \mathrm{~m}$ a.s.l. - RR - CR.

PUG, CAL, SIC.

TP: Mazara del Vallo presso C.da Critazzo (Troia PAL!, FI!), tra Roccolino sottano e Torre Grimes (Troia et al. PAL!), Roccolino (Troia \& Raimondo, 2009; Troia et al., 2012a; Troia \& Greuter, 2015b, c; Troia et al., 2019).

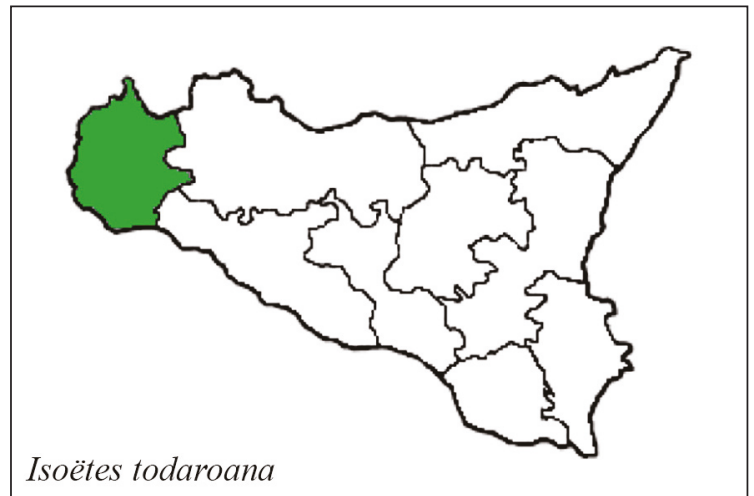

\section{Isoëtes longissima Bory}

Isoëtes velata var. longissima (Bory) A. Braun; I. velata A. Braun subsp. velata - Calamaria velata

I rad - SO-Steno-Medit.

Mediterranean temporary ponds, lives submerged (occasionally emerged) in lentic or weakly flowing fresh waters of seasonal wet environments, from 0 to 900 m a.s.l. - R - CR.

TOS, LAZ, PUG, SAR, SIC.

PA: Palermo al Piano Stoppa presso Misilmeri (?) (Todaro; Lojacono PAL!), Bosco e margi di Tumminia, Ficuzza presso il Lupo (PAL!), al piano stoppa presso Palermo (Todaro FI!), Bolognetta C.da Serralunga; TP: Pantani di Anguillara (Calatafimi Segesta) (Troia PAL!); ME: Messina; SR: Cozzo Ogliastro presso Sortino, Piana Sottana (Troia PAL!), Buccheri, nei pressi della stazione di Zelkova sicula, Piana Sottana, M. Lauro, Laghetto Francofonte scorrimento veloce CataniaRagusa, Bosco Pisano (Buccheri), Francofonte: Laghetto C.da Passanatello e Bosco Pisano (Giardina PAL!) (Lojacono, 1909; Raimondo et al., 1992; Marchetti, 2004; Troia, 2005; Giardina et al., 2007; Troia et al., 2012a; Troia \& Greuter, 2015b; Troia et al., 2016; Troia et Lansdown, 


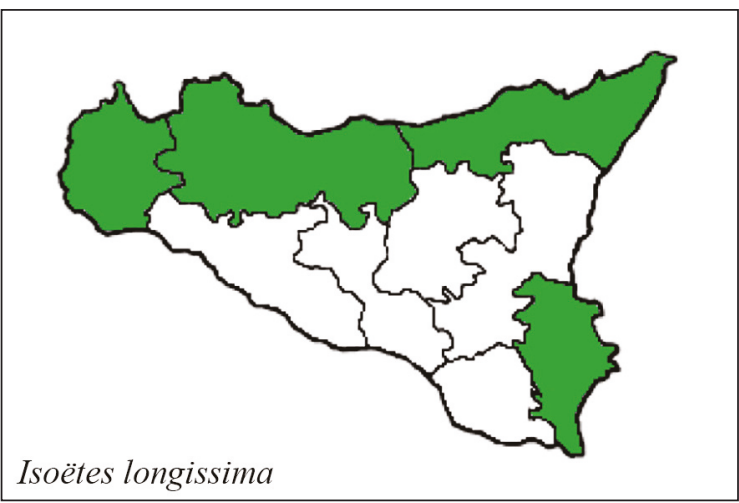

2016; Minissale \& al., 2017; Domina et al., 2019; Troia et al., 2019).

\section{SELAGINELLACEAE Willk.}

Genus Selaginella P. Beauv.

\section{Selaginella denticulata (L.) Spring}

Lycopodium denticulatum L. - Selaginella denticolata

Ch rept - Steno-Medit.-Macarones.

Escarpments and terraces, slopes, cliffs and walls, shaded and sunny, from 0 to $1500 \mathrm{~m}$ a.s.l. CC - LC.

LIG, TOS, MAR, LAZ, ABR, MOL, CAM, PUG, BAS, CAL, SAR, SIC.

\section{PA, TP, CT, ME, EN, AG, CL, RG, SR.}

Note. Selaginella kraussiana (Kunze) A. Braun is a tropical African species, naturalized in Macaronesia and Western Europe, that has also been reported in Sicily. Reported at the Etna (Fiori, 1943), escaped from the places of cultivation, but never in-

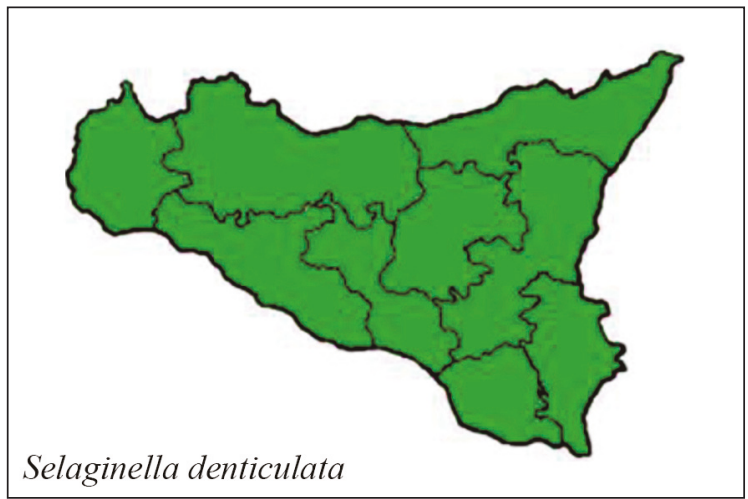

dependent in spreading (Marchetti, 2004; Giardina et al., 2007).

\section{EQUISETACEAE Michx ex DC}

\section{Genus Equisetum L.}

\section{Equisetum arvense L.}

Equiseto dei campi, Coda di cavallo dei campi.

G rhiz - Circumbor.

Marshes, puddles, waterways, torrent, streams, wet woods, uncultivated land, stones places, road margins, railways, from 0 to $2000 \mathrm{~m}$ a.s.1. - NC - LC.

FVG, VEN, TAA, LOM, PIE, VDA, LIG, EMR, TOS, MAR, UMB, LAZ, ABR, MOL, CAM, PUG, BAS, CAL, SAR, SIC.

PA: Fiume Oreto, Ficuzza, V.ne Reale, Riserva Pizzo Cane, Madonie (Minà PAL!), nei pressi di Polizzi Generosa, Castelbuono, S. Guglielmo (Di Martino PAL!), Margio Scorzone, V.ne Canna, V.ne della Trigna (Isnello) (PAL!), C.da Montaspro presso Isnello (PAL!), strada Pomieri-Geraci; TP: Alcamo, Mazzara del Vallo; ME: Capo d'Orlando (PAL!), Mistretta al fiume Santo Stefano, Messina (PAL!), Mirto (Citarda PAL!), Torrente Zappulla, Boschi di Valdemone (Todaro PAL!), Floresta in C.da Mitta, ruscelli di M. Scuderi (Giardina PAL!), Fiumara d' Agrò, V.ne Mandrazza (S. Lucia del Mela, M. Peloritani), V.ne Scala (PAL!), Fiumedinisi; CT: Boschetto della Plaja di Catania, Fontanazza di Adrano; EN: falde del M. Sambughetti, Riserva di Rossomanno-Grottascura-Bellia, M. Altesina; AG: Bivona in C.da Acque Bianche (Catanzaro PAL!), presso La Cava (Bivona); SR: Vendicari; RG: Marina di Modica, Acate a C.da Bosco Grande (Lojacono, 1909; Gramuglio et al., 1978; Raimondo et al., 1990; Rai-

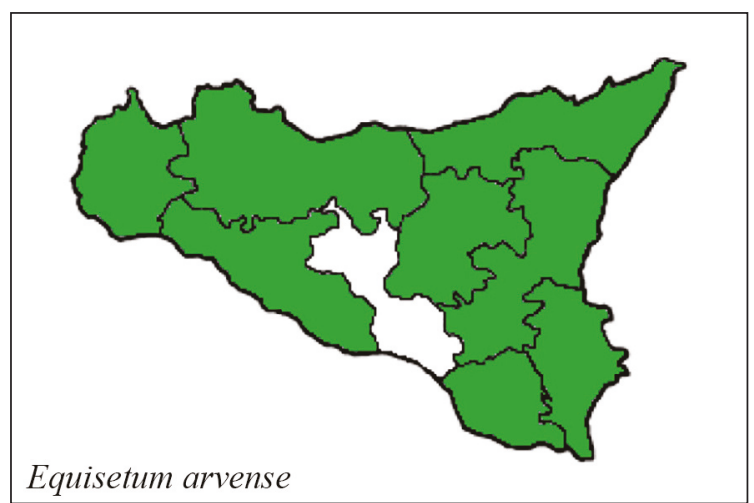


mondo et al., 2004; Luchino \& Grioli, 2016; Giardina et al., 2007; Domina et al., 2019).

\section{Equisetum palustre L.}

Equiseto palustre, Coda di cavallo palustre.

G rhiz - Circumbor.

Marshes, puddles, streams and damp ground, from 0 to $2000 \mathrm{~m}$ a.s.l. - RR - EN.

FVG, VEN, TAA, LOM, PIE, VDA, LIG, EMR, TOS, MAR, UMB, LAZ, ABR, MOL, CAM, PUG, BAS, CAL, SIC.

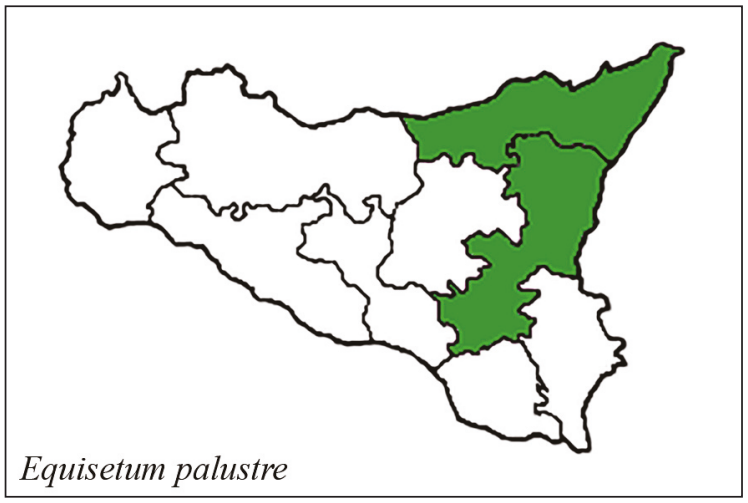

ME: Parco dei Nebrodi, Nebrodi a Portella Balestra, stagni del Bosco di Mangalavite (Bartolo FI!); CT: Arena di Catania, Valle del Flascio, Torrente Calanna, C.da Acquasanta, Gurna di Mascali (?) (Raimondo et al., 1992; Schicchi, 2004; Giardina et al., 2007).

\section{Equisetum ramosissimum Desf.}

Equisetum pallidum Bory; E. longevaginatum Strobl. - Equiseto ramosissimo, Coda di cavallo ramosissima

G rhiz - Paleotemp.

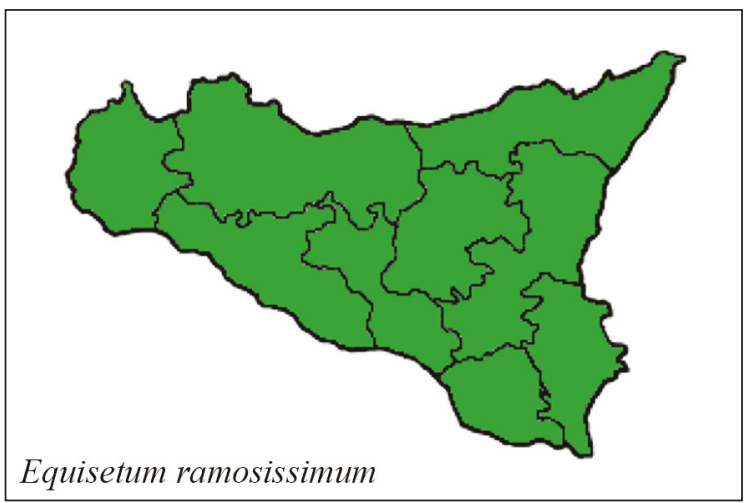

Stagnant or slow running waters, streams and damp ground, pebbles places, road margins, railways, etc. from 0 to $1500 \mathrm{~m}$ a.s.l. - CC - LC.

FVG, VEN, TAA, LOM, PIE, VDA, LIG, EMR, TOS, MAR, UMB, LAZ, ABR, MOL, CAM, PUG, BAS, CAL, SAR, SIC.

\section{PA, TP, CT, ME, EN, AG, CL, RG, SR.}

\section{Equisetum telmateia Ehrh.}

Equisetum telmateja Ehrh.; E. maximum Auct. Equiseto massimo, Equiseto maggiore, Coda di cavallo maggiore.

G rhiz - Euri-Medit-Macarones.

Marshes, puddles, streams, torrent, moist clayey meadows, from 0 to $1700 \mathrm{~m}$ a.s.1. - CC - LC.

FVG, VEN, TAA, LOM, PIE, VDA, LIG, EMR, TOS, MAR, UMB, LAZ, ABR, MOL, CAM, PUG, BAS, CAL, SAR, SIC.

PA, TP, CT, ME, EN, AG, CL, RG, SR.

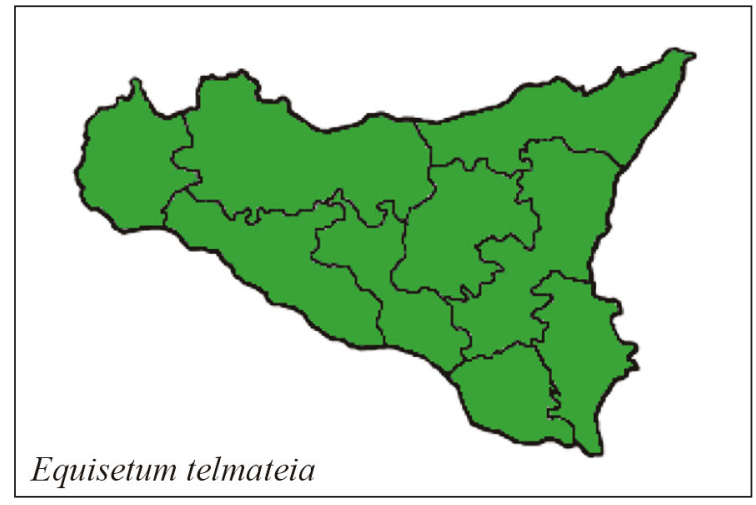

OPHIOGLOSSACEAE Martinov

Genus Botrychium Sw.

Botrychium lunaria (L.) Sw.

Osmunda lunaria L. - Botrichio lunaria

G rhiz - Orof.-Subcosmop.

Wet meadows, beech woods, from 1000 to 2000 m a.s.l. - RR - CR.

FVG, VEN, TAA, LOM, PIE, VDA, LIG, EMR, TOS, MAR, UMB, LAZ, ABR, MOL, CAM, PUG, BAS, CAL, SAR, SIC.

PA: Madonie a Montaspro, Sotto Polizzi 


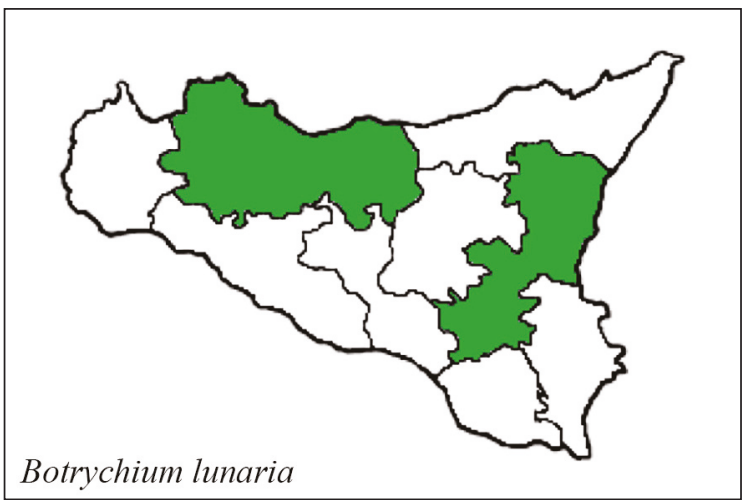

Generosa, Petralia Sottana alle Favare; CT: Etna (PAL!) (Gussone, 1845; Lojacono, 1909; Raimondo et al., 1992; Giardina et al., 2007).

\section{Genus Ophioglossum L.}

\section{Ophioglossum lusitanicum L.}

Ofioglosso lusitanico

G rhiz - Euri-Medit.-Subatlant.

Coastal meadows and wet clearings of the Mediterranean bush, slopes and earthy terraces, from 0 to $1000 \mathrm{~m}$ a.s.l. - NC - LC.

TOS, LAZ, CAM, PUG, CAL, SAR, SIC.

PA: Castellaccio, Mondello (Riccobono PAL!); TP: Isola di Pantelleria alle Fumarole di Favara Grande e M. Gibele, Trapani, M. Cofano, C.da Ballottella, C.da San Nicola (Mazara del Vallo), Isola Lunga dello Stagnone, da Trapani a Mazzara, Marsala (PAL!), da Capo Lilibeo al Ronciglio presso stazione di S. Teodoro (Marsala), S. Teodoro (Birgi), Calatafimi, Macchie di Alcamo (Reina PAL!), Gorghi Tondi, Birgi-S. Teodoro, Marsala nelle sciare (PAL!), Mazzara del Vallo, Pietra Colle (Buseto Palizzolo), Bosco di Birribaida (Castelve-

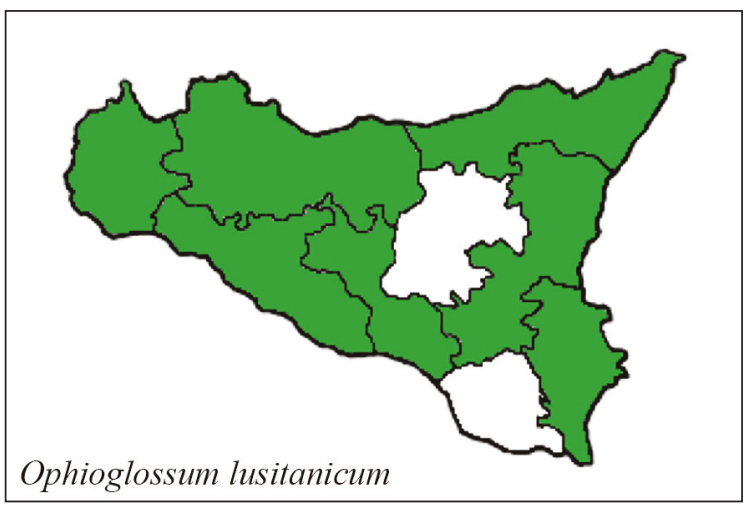

trano), pantani di Contrada Anguillara (Calatafimi); ME: Isola di Vulcano cratere "La Fossa", Alicudi, Colli di Messina (PAL!), Terranova a Dorillo, Castanea delle Furie (PAL!), Castroreale e Colle Catalano, Gravitelli, Foresta Camaro, tra Camaro e Caterratte, Torrente Agro', Fiume Alcantara M. Ciccia; CT: Catania (Citarda PAL!), Nicolosi, Acicatena, Caltagirone a S. Pietro, Pantano Catania, dal Fiume Simeto a Paternò, Tre Monti, M. Ciccia, Etna a M. Po, Etna colata del 1651, San Giovannello, Fleri (frazione del comune di Zafferana Etnea), Pennina di Lupo, Belpasso; AG: Isola di Linosa (Sommier PAL!), Isola di Linosa presso Mannarazza (Sommier PAL!), Lago Arancio; CL: Niscemi, Biviere Gela; SR: S. Corrado, Casazza, scogliere di Capo Passero (Gussone, 1845; Lojacono, 1909; Gianguzzi, 2003; Aleo et al., 2004; Raimondo et al., 2004; Gianguzzi et al., 2005; Giardina et al., 2007; Legambiente, 2009; Troia et al., 2012b; Médail et al., 2016; Domina et al., 2019).

\section{Ophioglossum vulgatum $\mathrm{L}$.}

Ophioglossum alpinum Rouy; O. vulgare L. - Ofioglosso comune

G rhiz - Circumbor.

Wet and shady meadows, marshes, streams, shady beech woods, from 0 to $1700 \mathrm{~m}$ a.s.l. - RR - EN.

FVG, VEN, TAA, LOM, PIE, LIG, EMR, TOS, MAR, UMB, LAZ, ABR, MOL, CAM, BAS, CAL, SAR, SIC.

PA: Madonie (Citarda PAL!), Madonie alle Favare di Petralia Sottana (Giardina PAL!), nei pressi di Polizzi Generosa; ME: Nebrodi a M. Soro (Todaro PAL!), Peloritani alle Serre di Cannata, Monti di Lercara in Valdemone (Todaro PAL!) (Lojacono,

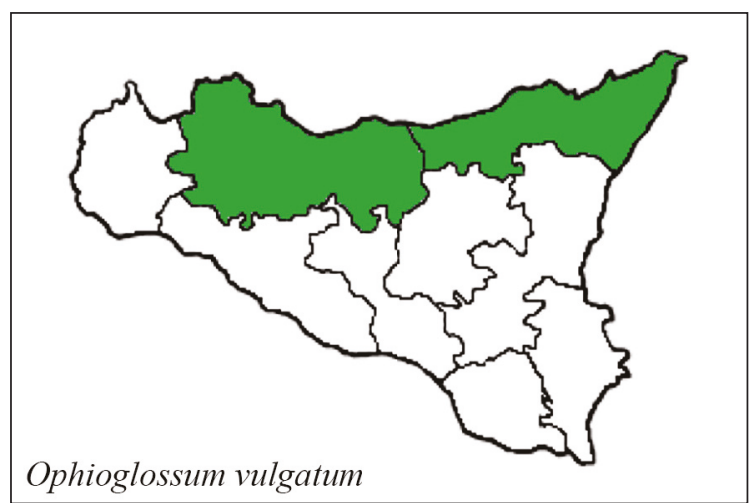


1909; Raimondo et al., 1992; Raimondo et al., 2004; Giardina et al., 2007).

\section{OSMUNDACEAE Martinov}

Genus Osmunda L.

\section{Osmunda regalis L.}

Osmunda regale, Felce florida

G rhiz - Subcosmop.

Mountain whirlpools, marshes, streams, humid woods, from 0 to $1200 \mathrm{~m}$ a.s.l. - R - VU.

VEN, TAA?, LOM, PIE, LIG, EMR, TOS, MAR?, LAZ, CAL, SAR, SIC.

PA: Monti di Palermo a Punte dei Cuti, Mezzojuso al V.ne Cerasa, Partinico, Ficuzza al V.ne Arcera, Ficuzza al V.ne Cerasa e Ficuzza al V.ne della Castagnera, Madonie (Lojacono; Todaro PAL!), Castelbuono (Minà PAL!), Castellobuono nel V.ne del Canalicchio (Ross PAL!), Madonie al V.ne Scopalacqua, Madonie al V.ne sotto Rocca di Mele (Ross PAL!), Collesano, Margio Scorzone, Fridda, Vicaretto, strada Pomieri-Geraci, Piano Pomo, Gorgo Nero di Petralia Sottana; ME: Messina ai Gammari e V.ni di Piano del Campo, Messina al Camaro, Catarratti, al Campo, C.da Scala (PAL!), S. Miceli, Salice, Serro Paglierotto, Itala, Antillo, M. Ciccia, V.ne sotto M. Scuderi (Raimondo et al. PAL!), M. Scuderi presso Punt.le Puzzu (Raimondo PAL!), Rometta a Rocche Jero (Peloritani), S. Stefano Briga a S. Calorio, Pellegrino alla Sorgente Imbardo, Fiumedinisi, Mandanici, extinct at Vulcano (Aeolian islands); CT: Etna(?) (Bivona? PAL!), Caltagirone a Noce Paradiso (Gussone, 1845; Lojacono, 1909; Picone et al., 2003; Gianguzzi \& La Mantia, 2004; Raimondo et al., 2004; Giardina et al., 2007; Domina et al., 2019).

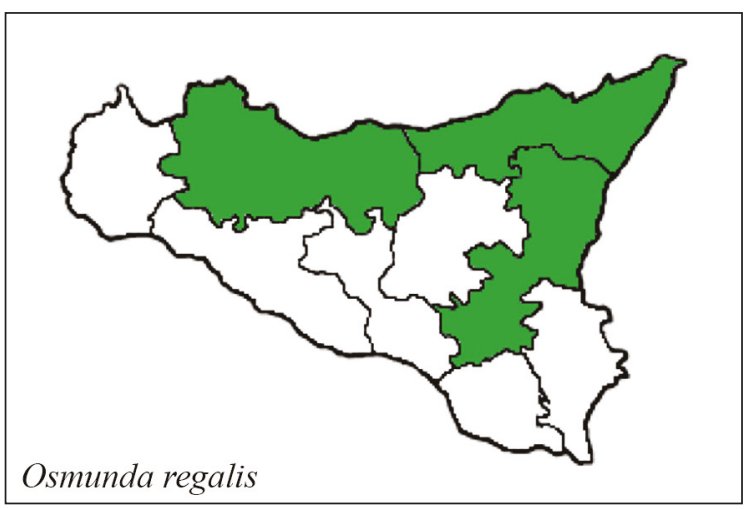

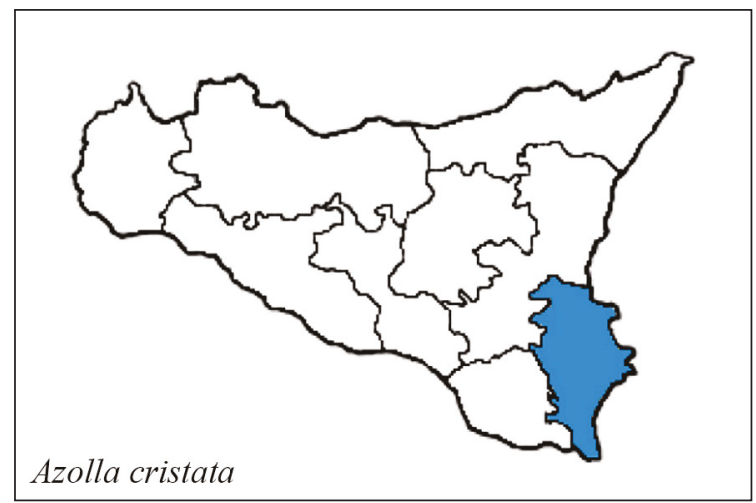

SALVINIACEAE Martinov

Genus Azolla Lam.

\section{Azolla cristata Kaulf.}

Azolla caroliniana Auct. non Willd.; A. mexicana C. Presl - Azolla crestata

I nat $/ T$ - Neotropic. Invasive species.

Stagnant or slow-flowing waters, from 0 to 300 m a.s.l. - RR

SIC.

SR: Lungo il Fiume Ciane; Parco archeologico di Neapolis (Siracusa) (Romano et al., 1994; Giardina et al., 2007; Minissale \& Sciandrello, 2017; Troia et al., 2020).

\section{Azolla filiculoides Lam.}

Azolla caroliniana Willd.; A. mexicana C. Pres1 Azolla maggiore, Azolla americana

I nat/ $\mathrm{T}$ - Neotropic. Invasive species.

Stagnant or slow-flowing waters, from 0 to 300 $m$ a.s.l. - RR

VEN, TAA, LOM, PIE, EMR, TOS, MAR, UMB, LAZ, CAM, PUG, BAS, CAL, SAR, SIC.

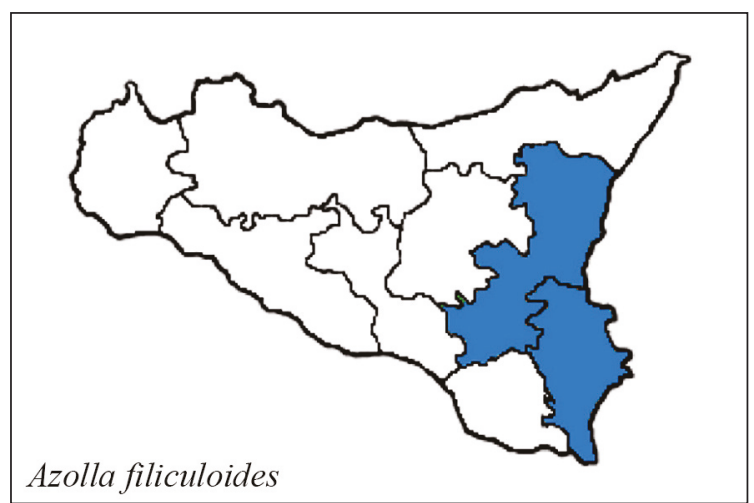


CT: Pantano Gurna di Mascali? [Maybe disappeared for the reclamation of this area], foce del torrente Fiumefreddo, Acireale; SR: Fiume Ciane, Parco archeologico di Neapolis (Siracusa) (Romano et al., 1994; Giardina et al., 2007; Albano, 2010; Giordana, 2013; Minissale \& Sciandrello, 2017; Troia et al., 2020).

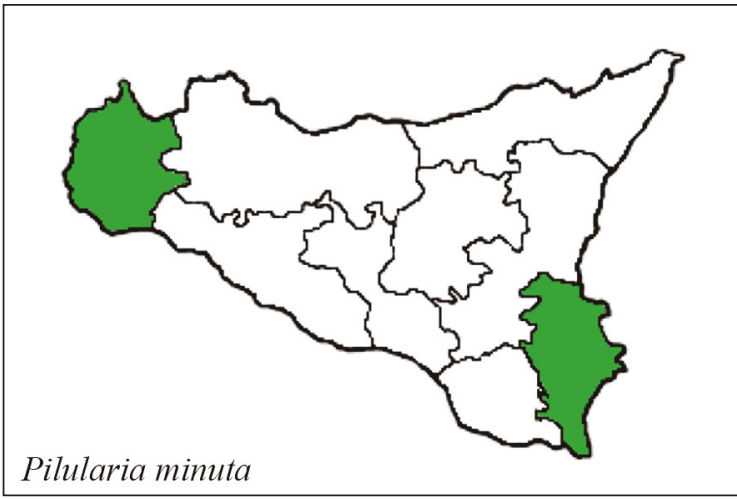

MARSILEACEAE Mirb.

Genus Pilularia L.

Pilularia minuta Durieu ex Braun

Pilularia minore

I rad/G rhiz - W-Steno-Medit.

Stagnant waters and ephemeral pools, from 0 to $600 \mathrm{~m}$ a.s.l. - RR - EN.

LAZ, PUG, SAR, SIC.

TP: Trapani presso Bonagia (Nicotra FI!) (?), Pantani di Anguillara (Calatafimi Segesta); SR: Cozzo Fico (Carentini), Cozzo Ogliastri (Sortino) (Lojacono, 1909; Marchetti, 2004; Giardina et al., 2007; Troia et al., 2016; Troia \& Lansdown, 2016; Minissale et al., 2017).

PTERIDACEAE E. D. M. Kirchn

\section{Genus Adiantum L.}

\section{Adiantum capillus-veneris L.}

Capelvenere Comune

G rhiz - Pantropic./Subtropic.

Wet cliffs, walls, caves, springs, from 0 to 1500 m a.s.l. - CC - LC.

FVG, VEN, TAA, LOM, PIE, VDA, LIG, EMR,

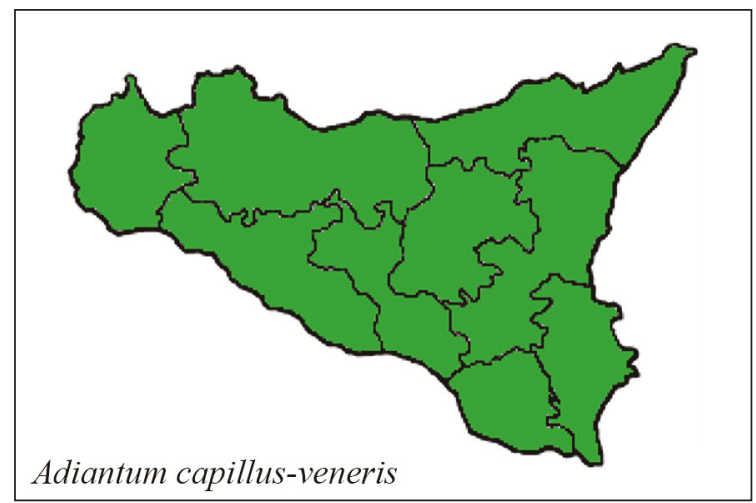

TOS, MAR, UMB, LAZ, ABR, MOL, CAM, PUG, BAS, CAL, SAR, SIC.

PA, TP, CT, ME, EN, AG, CL, RG, SR.

Genus Anogramma Link

Anogramma leptophylla (L.) Link

Polypodium leptophyllum L.; Gymnogramme leptophylla Desv; Grammitis leptophylla (L.) Sw. Felcetta annuale, Anogramma a foglie sottili

T caesp - Pantropic.

Rock crevices, shady and humid walls, from 0 to $1300 \mathrm{~m}$ a.s.1. - CC - LC.

VEN, TAA, LOM, PIE, VDA, LIG, EMR, TOS, LAZ, MOL, CAM, PUG, BAS, CAL, SAR, SIC.

PA, TP, CT, ME, EN, AG, CL, RG, SR.

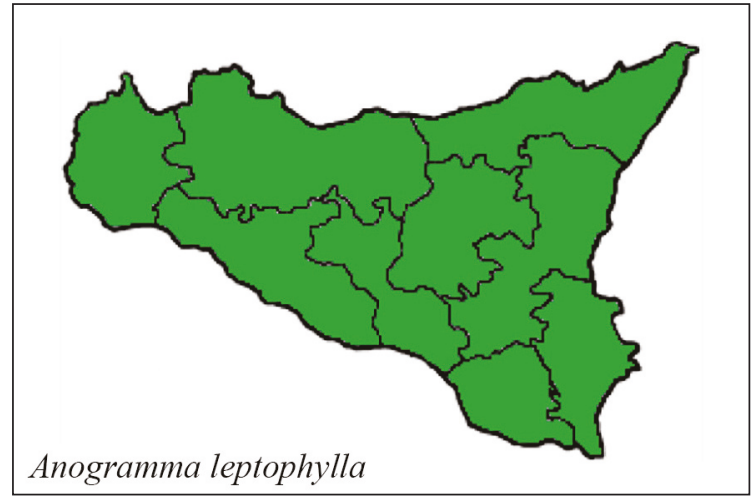

Genus Cheilanthes Sw.

Cheilanthes acrostica (Balb.) Tod.

Pteris acrostica Balb.; Hemionitis acrostica (Balb.) Mosyakin; Allosorus acrosticus (Balb.) Christenh.; Cheilanthes pteridioides (Reichard) C. Chr.; Ch. pteridioides subsp. acrostica (Balb.) O. Bolòs et al.; 


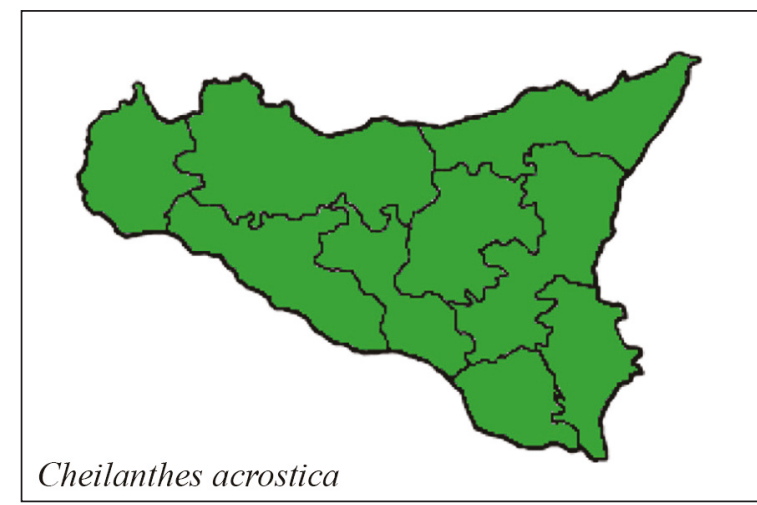

Ch. odora Sw.; Ch. fragrans Sw.; Oeosporangium acrosticum (Balb.) L. Sáez \& Aymerich. - Felcetta odorosa

H ros - Steno-Medit.-Turan.

Cliffs and walls, from 0 to $1100 \mathrm{~m}$ a.s.1. - CC LC.

PIE, VDA, LIG, TOS, LAZ, CAM, PUG, CAL, SAR, SIC.

PA, TP, CT, ME, EN, AG, CL, RG, SR.

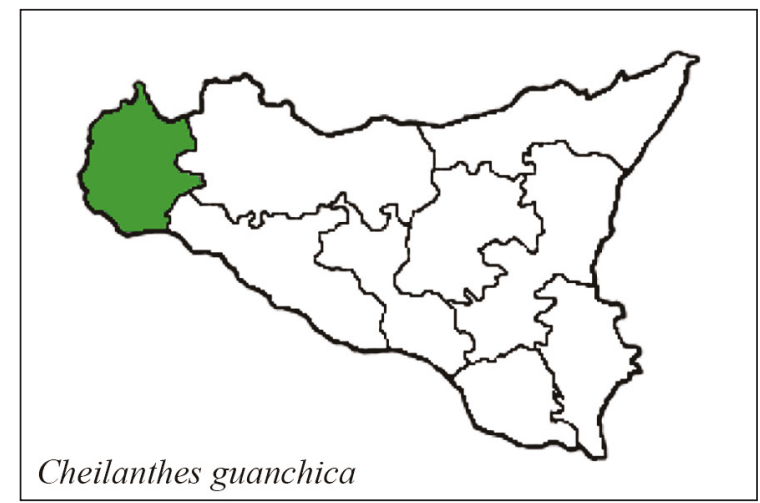

Cheilanthes guanchica Bolle

Cheilanthes pteridioides subsp. guanchica (Bolle) O. Bolòs et al.; Allosorus guanchicus (Bolle) Christenh.; Oeosporangium guanchicum (Bolle) FraserJenk. \& Pariyar - Felcetta delle Canarie

H ros - Steno-Medit.-Macarones. VU.

Cliffs and walls, from 0 to $570 \mathrm{~m}$ a.s.1. - R -

LIG?, TOS?, SAR, SIC.

TP: Pantelleria presso Fossa del Russo (CAT!) (Marchetti et al., 2008; Troia et al., 2012b).
Cheilanthes maderensis Lowe

Polypodium pteridioides Reichard; Allosorus pteridioides (Reichard) Christenh.; Oeosporangium pteridioides (Reichard) Fraser-Jenk. \& Pariyar (see also above) - Felcetta di Madera

H ros - Steno-Medit.-Macarones.-Sahar.

Cliffs and walls, from 0 to $600 \mathrm{~m}$ a.s.1. - R - VU. LIG, TOS, LAZ?, CAM, PUG, CAL, SAR, SIC.

PA: Palermo (Domina PAL!), M. Pellegrino, Castellaccio presso Monreale (Roccobono PAL!), M. Catalfano, M. Irice (PAL!), Castelbuono presso Piano S. Paolo (PAL!), Castelbuono nel Castello, dirupi sotto Isnello (PAL!), rupi di Pizzo di Pilo (PAL!); TP: Pantelleria a Montagna Grande, Siba (Bartolo; P. Minissale \& G. Spampinato CAT!), M. Gibele, Fossa del Russo (Bartolo; P. Minissale \& G. Spampinato CAT!), Bugeber, Rakhale, M. Gelkhamar, Lave Gelfizer, Cuddia Attalora, Isola di Marettimo, Calatafimi (?), Zingaro alla Tonnarella dell'Uzzo presso Torre dell'Uzzo (Raimondo PAL!), M. Cofano (?); ME: Isole di Alicudi, Filicudi (CAT!), Panarea (CAT!), Salina CAT!), Tindari presso i Laghetti di Marinello, Malfa, S. Margherita, Galati Marina, Castelmola, Capo S. Andrea, Baia Isola Bella, rupi di Taormina, M. Veneretta, Madonna

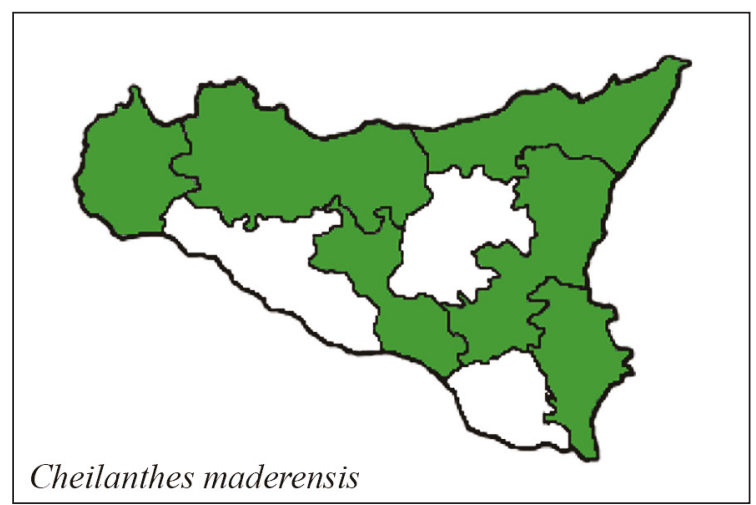

della Rocca (Taormina); CT: Chiuse di Catania (?), Acireale, Massannunziata, Tra Mascalucia e Nicolosi, M. La Nave (C. Barbagallo \& C. Scalisi CAT!), Etna, Colata lavica del 1381 (M. Grillo \& E. Poli CAT!), M. Ciraulo, Collina di Monte Po; CL: Sutera presso M. S. Paolino (Reina PAL!); SR: Strada Sortino-Ferla (Raimondo et al., 1992; Gianguzzi, 2003; Marchetti, 2004; Turrisi, 2004; Minissale et al., 2005; Gianguzzi et al., 2005, 2006; Giardina et al., 2007; Marchetti et al., 2008; Giardina, 2010; Licandro et 
al., 2011; Troia et al., 2012(b); Sciandrello et al., 2013, 2014; Domina et al., 2019).

Cheilanthes tinaei Tod.

Cheilanthes corsica Reichst. \& Vida; Ch. pteridioides subsp. tinaei (Tod.) O. Bolòs \& al.; Allosorus tinaei (Tod.) Christenh.; Oeosporangium tinaei (Tod.) Fraser-Jenk. - Felcetta di Corsica, Felcetta di Tineo

H ros - Steno-Medit.-Macarones.

Cliffs and walls, from 0 to $500 \mathrm{~m}$ a.s.1. - R - VU. LIG, TOS, CAL, SAR, SIC.

PA: Palermo, Monreale, Arenella; ME: Isole di Alicudi (CAT!), Filicudi, Panarea (CAT!), Vulcano, Salina tra Arenella e Val di Chiesa (Lojacono CAT!), Malfa, Messina (PAL!), Milazzo, Francavilla di Sicilia (Peloritani), Scaletta Zanclea (P. Minissale \& G. Spampinato CAT!), Mili, S. Margherita, Giampilieri, M. Scuderi, Mandanici, Artolia, Tremestieri, Saponara, Francavilla, Taormina, Rinella (Leni); CT: Chiuse di Catania, Milo, Acireale, Misterbianco, Alcantara, S. Tecla, Bongiardo, Acicatena, V.ne Ulli, S. Alfio; AG: Montaperto (Lojacono, 1909; Raimondo et al., 1992; Marchetti, 2004; Raimondo et al., 2004; Giardina et al., 2007; Marchetti et al., 2008; Troia et al., 2012(b); Sciandrello et al., 2014; Domina et al., 2019).

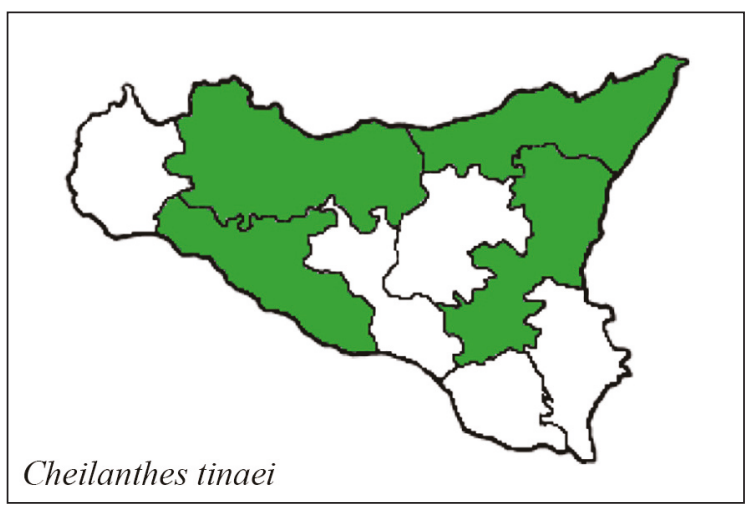

Genus Cosentinia Tod.

Cosentinia vellea (Aiton) Tod. subsp. vellea Acrosticum velleum Aiton; $A$. lanuginosum Desf.; $A$. catanense Cosent.; Notholaena vellea (Aiton) Desv.; $N$. lanuginosa (Desf.) Poir.; N. lanuginosa (Desf.) Poir. subsp. lanuginosa; Cheilanthes catanensis (Cosent.) Fuchs; $C$. vellea (Aiton) F. Muell.; C. vellea (Aiton) F. Muell. subsp. vellea. - Felcetta catanese

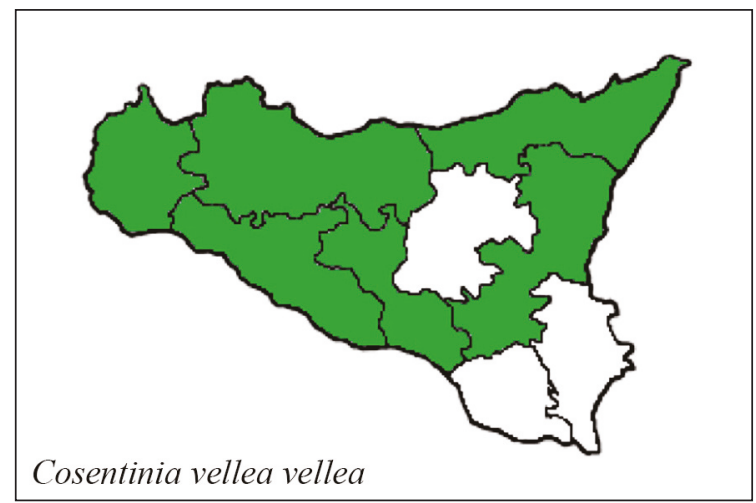

H ros - Macarones.

Cliffs and sunny walls from 0 to $500 \mathrm{~m}$ a.s.1. $\mathrm{R}$ - LC.

TOS, CAM?, PUG, BAS?, CAL?, SAR, SIC.

PA: Rupi calcaree di Palermo (Todaro PAL!), Palermo alla Rocca sopra la Catena (PAL!), rupi di M. Pellegrino (Todaro PAL!), M. Pellegrino a Villa Belmonte (Riccobono PAL!), Rocca, M. Gallo lato Tommaso Natale, San Giuseppe, rupi di M. Catalfano (G. Domina; Todaro; Lojacono PAL!), M. Irice (PAL!), Capo Zafferano, Solunto (G. Certa \& G. Scafidi PAL!), C.da Cavallo di Mezzo (M. Catalfano) (Certa PAL!); TP: Isola di Pantelleria (Todaro PAL!) (Gelfizer, Gelkhamar, Rakhali, Fossa del Russo Bugeber, Khafar, Mursia), Isola di Marettimo, Isola di Levanzo, Castellammare in C.da Fraginesi; ME: Isole di Salina al V.ne Olivo Grande, Linosa a M. di Ponente e M. Vulcano (Sommier PAL!), Capo Tindari, Salita di Scaletta, Fiumedinisi, Graniti, Mandanici, rupi di Taormina, Madonna della Rocca (Taormina), Capo Taormina; CT: Catania (PAL!), tra Acireale e S. Tecla, Catania al Parco dei Gioeni, rupi delle Chiuse di Catania (PAL!), Etna colata del 1381 al Canalicchio; CL: Caltanissetta (PAL!); AG: Linosa a M. di Ponente e M. Vulcano (Sommier PAL!), Cala Pozzolana di Levante (Linosa) (Gussone, 1845; Lojacono, 1909; Raimondo et al., 1992; Raimondo et al., 1993; Gianguzzi, 2003; Marchetti, 2004; Raimondo et al., 2004; Gianguzzi et al., 2006; Romano et al., 2006; Giardina et al., 2007; Gristina \& Marcenò, 2008; Giardina, 2010; Marchetti et al., 2010; Troia et al., 2012b; Sciandrello et al., 2013; Federico, 2015; Domina et al., 2019).

Cosentinia vellea (Aiton) Tod. subsp. bivalens (Reichst.) Rivas Mart. \& Salvo

Acrosticum velleum Aiton; A. lanuginosum Desf.; A. catanense Cosent.; Notholaena vellea (Aiton) 


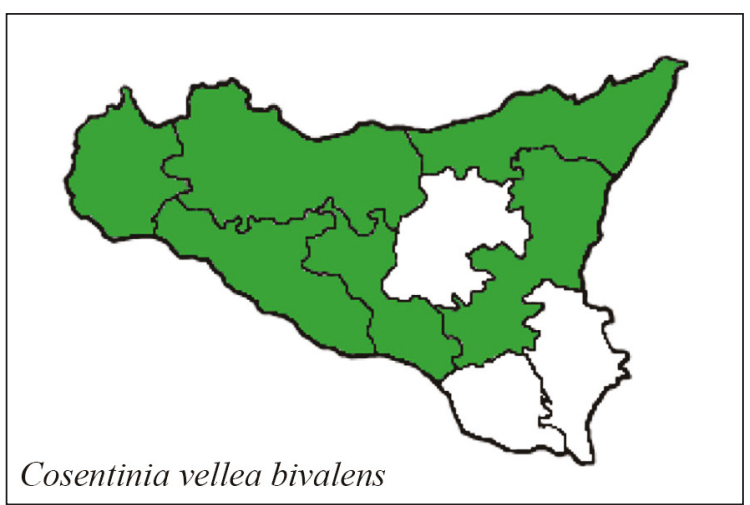

Desv.; N. lanuginosa (Desf.) Poir.; N. lanuginosa (Desf.) Poir. subsp. bivalens Reichst.; Cheilanthes catanensis (Cosent.) Fuchs; C. vellea (Aiton) F. Muell.; C. vellea (Aiton) F. Muell. subsp. bivalens. (Reichst.) Greuter \& Jermy. - Felcetta bivalente

H ros - Medit.-Turan

Cliffs and sunny walls from 0 to $500 \mathrm{~m}$ a.s.1. $\mathrm{R}$ - LC.

SAR, SIC.

PA: M. Cofano presso C.da Cavallo di Mezzo (Certa PAL!), Capo Zafferano, M. La Città (comune di S. Flavia); TP: Castellammare del Golfo, Pantelleria, M. Cofano, Isole Egadi; ME: Capo Tindari (CAT!), Laghetti di Marinello, Taormina (CAT!); CT: Catanese; AG: Linosa; CL: Caltanissetta; (Marchetti et al., 2008; Marchetti et al., 2010; Licandro et al., 2011; Sciandrello et al., 2014; Domina et al., 2019).

\section{Genus Pteris L.}

Pteris cretica L.

Pteris olygophylla Viv. - Pteride di Creta

H ros - Pantropic.

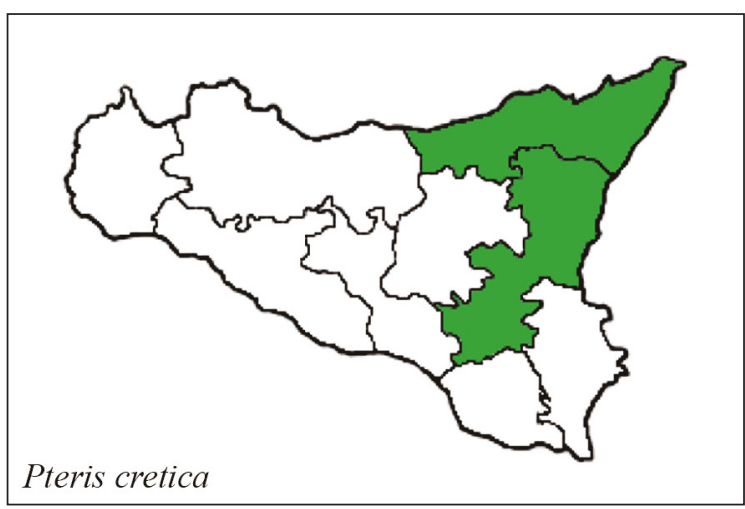

Shady cliffs, streams, humid and shady woods, cracks and cliffs, from 0 to $800 \mathrm{~m}$ a.s.l. - R - EN.

FVG, VEN, TAA, LOM, PIE, LIG, TOS, LAZ, ABR?, CAM, SAR, SIC?

ME: Fra Milazzo e Spadafora, Giampilieri, Fiumedinisi, Mandanici, S. Margherita, Taormina; CT: Etna al Milo (PAL!) (Gussone, 1845; Lojacono, 1909; Raimondo et al., 1992; Giardina et al., 2007; Sciandrello et al., 2014). Not recently reported.

\section{Pteris vittata L.}

Pteris longifolia Auct. - Pteride a foglie lunghe

H ros - Subcosmop.

Cliffs and walls, humid and shady environments, from 0 to $400 \mathrm{~m}$ a.s.1. - R - VU.

CAM, CAL, SIC. Naturalized in VEN, PIE, LIG, UMB, LAZ.

PA: Bosco Ficuzza; ME: Peloritani, luoghi selvatici presso Messina (Todaro PAL!), Milazzo (Citarda PAL!), tra Galati e Santo Stefano, Fiumedinisi, Giardini, Giampelieri, Fiumara di Mandanici (PAL!), S. Margherita, Taormina, Merì, presso abitato di Fadarechi di Casalvecchio Siculo, torrente Portalegni; SR: M. Iblei, fiume Anapo e fiume Ciane, V.ne di Melilli (Tin. PAL!), Melilli alla Fontana dell'Orto del Monaco (PAL!), Siracusa corsi d'acqua dei Mulini dell'Anfiteatro (PAL!), Cava Grande del Cassibile, Avola (Bianca PAL!), Avola a Cava dell' Amico, Riserva Naturale Villasmundo - S. Alfio; RG: Cava d'Ispica (Gussone, 1845; Lojacono, 1909; Raimondo et al., 1992; Marchetti, 2004; Giardina et al., 2007; Giardina, 2010; Sciandrello et al., 2014; Domina et al., 2019).

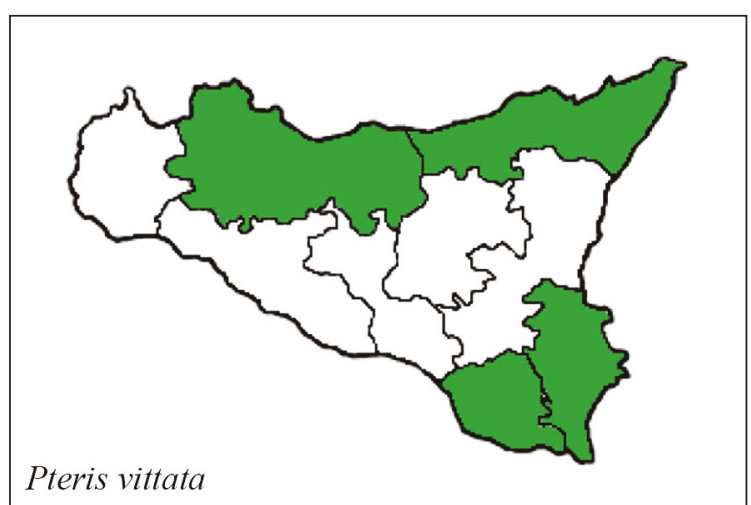




\section{DENNSTAEDTIACEAE Lotsy}

Genus Pteridium Gled. ex Scop.

Pteridium aquilinum (L.) Kuhn subsp. aquilinum Pteris aquilina L. - Felce aquilina

G rhiz - Cosmop.

Sparse woods, bushes, uncultivated, pastures, from 0 to $2100 \mathrm{~m}$ a.s.1. - CC - LC.

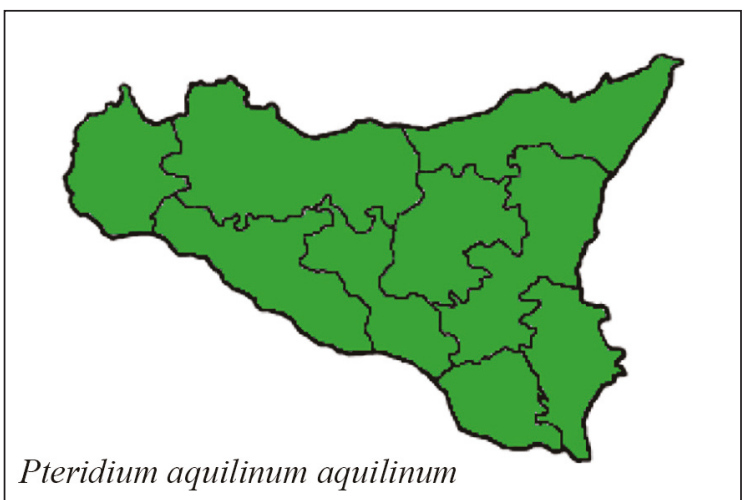

FVG, VEN, TAA, LOM, PIE, VDA, LIG, EMR, TOS, MAR, UMB, LAZ, ABR, MOL, CAM, PUG, BAS, CAL, SAR, SIC.

PA, TP, CT, ME, EN, AG, CL, RG, SR.

\section{CYSTOPTERIDACEAE Shmakov}

Genus Cystopteris Bernh.

Cystopteris alpina (Lam.) Desv.

Polypodium alpinum Lam.; Cystopteris fragilis subsp. alpina (Lam.) Hartman; C. regia Auct. - Felcetta alpina

H caesp - Artico-Alp. (Europ.).

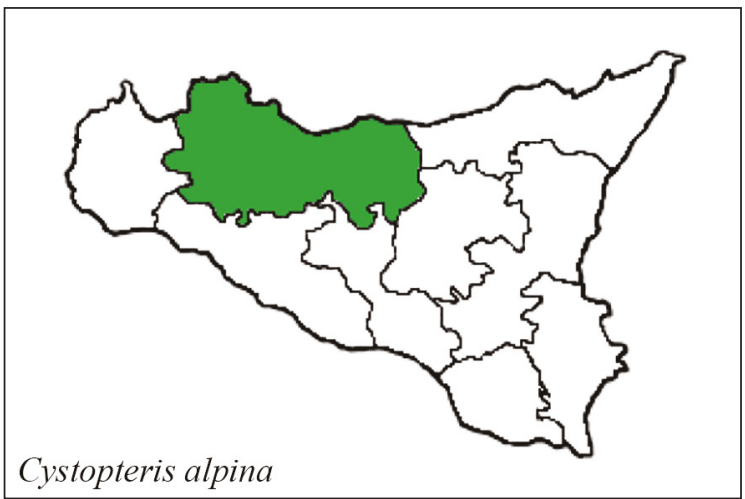

Cliffs, rock cracks and scree, in humid and shady environments, from 1000 to $1800 \mathrm{~m}$ a.s.l. RR - EN.

FVG, VEN, TAA, LOM, PIE, VDA, LIG, EMR, TOS, MAR, UMB?, LAZ, ABR, MOL, CAM, BAS, CAL, SIC.

PA: Madonie, vicino Castelbuono (Lojacono, 1909; Marchetti, 2004; Raimondo et al., 2004; Giardina et al., 2007).

\section{Cystopteris dickieana R. Sim}

Cystopteris baenitzii Dorfl.; C. regia (L.) Desv. var. dickieana (R. Sim) Druce.; Cystopteris fragilis subsp. dickieana (R. Sim) Hyl. - Felcetta dickieana, Felcetta di Dickie

H caesp - Subcosmop.

Cliffs, rock cracks and scree, in humid and shady environments, from 400 to $2500 \mathrm{~m}$ a.s.1. - NC - VU.

TAA, LOM, PIE, VDA, EMR?, TOS?, LAZ, ABR, CAL, SAR, SIC.

PA: Rocca Busambra, Madonie (Tornabene

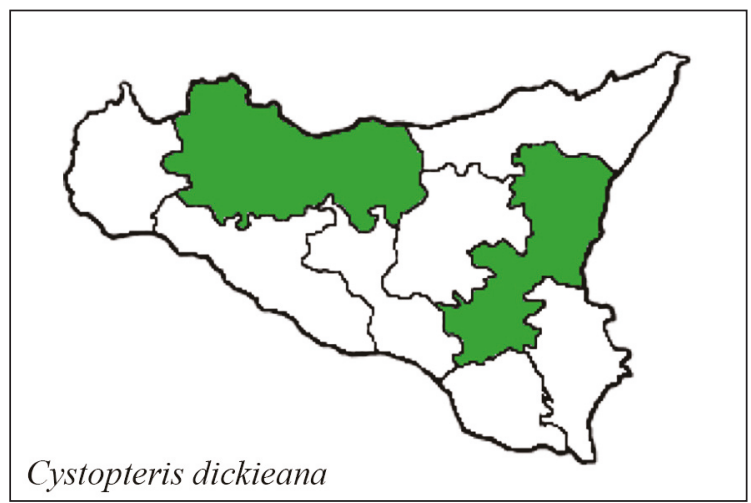

CAT!; Todaro TO!), Madonie al Passo della Botte (G. Scafidi \& E. Schimmenti PAL!); CT: Etna alla Casa degli Inglesi (Huet du Pavillon FI! e TO!) e M. Albano, Nicolosi, Catania, Ognina (Tornabene CAT!) (Lojacono, 1909; Marchetti, 2004; Giardina et al., 2007).

Cystopteris fragilis (L.) Bernh.

Polypodium fragile L.; Cystopteris canariensis C. Presl - Felcetta fragile

H caesp - Cosmop.

Cliffs, rock cracks and scree, caves, in humid 


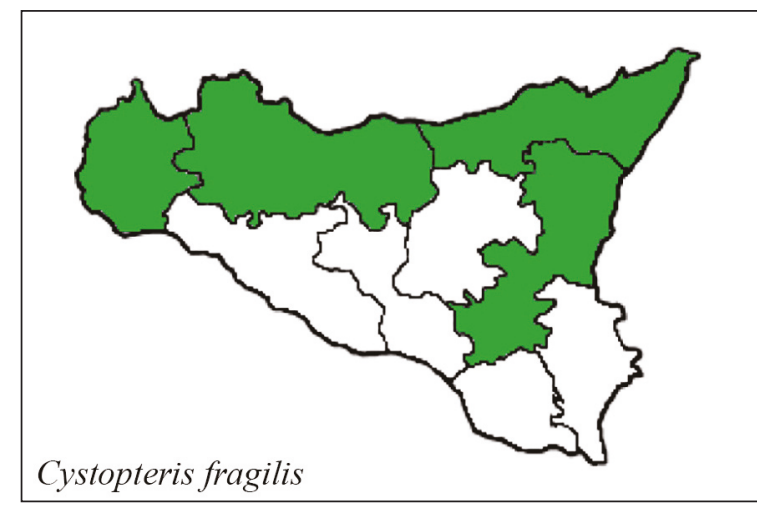

and shady environments, from 100 to $3000 \mathrm{~m}$ a.s.l. - C - LC.

FVG, VEN, TAA, LOM, PIE, VDA, LIG, EMR, TOS, MAR, UMB, LAZ, ABR, MOL, CAM, PUG, BAS, CAL, SAR, SIC.

PA: Madonie (Tineo; Todaro PAL!), Madonie vicino Castelbuono, Manche di Caltavuturo (PAL!), presso La Sciara di Caltavuturo (PAL!), V.ne del Passo della Botte (Ross PAL!), V.ne Fra Paolo presso Petralia Sottana (PAL!), Madonie ai Boschi del M. Salvatore (Ross PAL!); TP: Isola di Pantelleria; ME: Lipari, Valdemone (Citarda PAL!), Nebrodi e Peloritani, Salice, Antennammare, Caronia (Reina PAL!); CT: Etna (PAL!), Acireale, Etna a M. Silvestri e M. del Salifizio, Nicolosi, presso La Ginestra (PAL!), Milo presso Crateri del 1928 (Sant'Alfio) (PAL!), Etna presso C.da Previtera a Zafferana Etnea (PAL!) (Raimondo et al., 2004; Giardina et al., 2007; Gianguzzi, 2003; Domina et al., 2019).

\section{ASPLENIACEAE Newman}

\section{Genus Asplenium L.}

\section{Asplenium balearicum Shivas}

Asplenio delle Baleari

H scap - NO-Steno-Medit.

Rock cracks and cliffs, from 0 to $100 \mathrm{~m}$ a.s.l. RR - EN.

TOS, LAZ, SAR, SIC.

PA: Ustica (PAL!), Castelbuono ai Monticelli (Giardina; Minà PAL!); TP: Isola di Pantelleria (Giardina PAL!), Pantelleria a Mandra di Gallo vi-

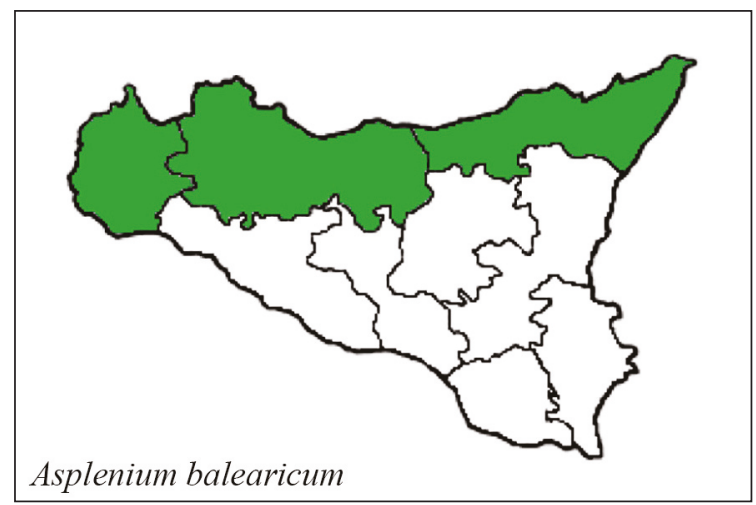

cino la Stufa di Monastero presso Scauri (PAL!), Cuddie Rosse/Gelkhamar, Punta Fram, lave, presso Scauri e Khaggiar (Giardina PAL!), Piano di ghirlanda (FI!); ME: Alicudi, Messina (Giardina PAL!); AG: Extinct in Lampedusa near V.ne Imbriacola (PAL!) (Gianguzzi, 1999; Raimondo et al., 1992; Gianguzzi, 2003; Marchetti, 2004; Giardina et al., 2007; Marchetti et al., 2009; Troia et al., 2012b; Domina et al., 2019).

\section{Asplenium lepidum C. Presl subsp. lepidum \\ Asplenio grazioso}

H ros - Orof. SO-Europ.

Cracks and limestone, shady and humid walls, from 200 to $2250 \mathrm{~m}$ a.s.1. - RR - VU.

LOM, PIE?, VEN, FVG, TAA, MAR, ABR, LAZ, CAM, BAS, CAL, SIC.

PA: Dalle Madonie a Palermo, M. Gallo (PAL!), Rocca Busambra (PAL!), Rocca Busambra alle Neviere, Ficuzza (PAL!), Madonie (Tineo PAL!), Madonie al Balzo Canna (PAL!), rupi di Isnello (PAL!); ME: Nebrodi (Lojacono PAL!); CT: Etna (Lojacono PAL!). (Lojacono, 1909; Marchetti, 2004; Giardina et al., 2007; Domina et al., 2019).

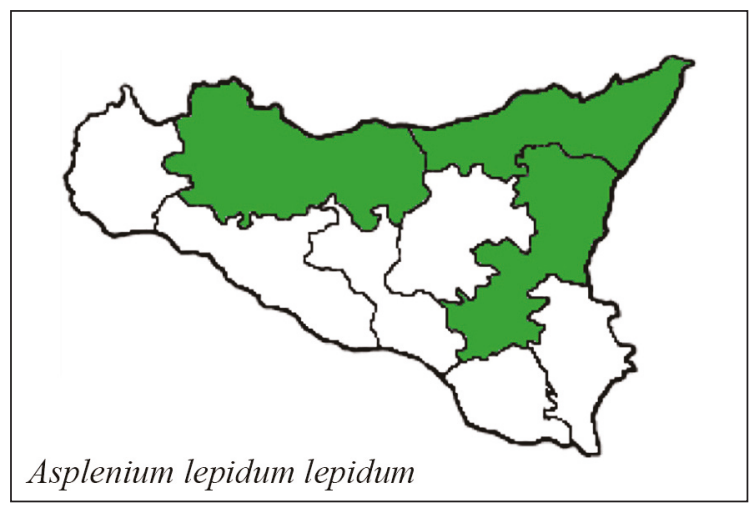




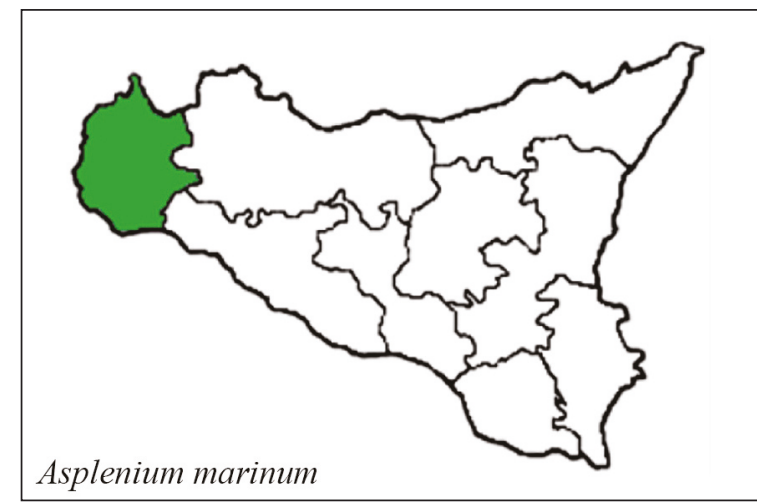

Asplenium marinum L.

Asplenio marino

H ros - SW-Europ. (Subatlant.).

Rock and walls, by the sea, from 0 to $830 \mathrm{~m}$ a.s.l. - RR - CR.

TOS, LAZ, PUG, SAR, SIC.

TP: Isola di Pantelleria (Lojacono; Errera; Citarda PAL!), Pantelleria a M. Grande, Gelkhamar, vicino la Grotta del Mordome (Errera PAL!) (Gussone, 1845; Lojacono, 1909; Gianguzzi, 1999, 2003; Raimondo et al., 1992; Marchetti, 2004; Giardina et al., 2007; Troia et al., 2012b; Domina et al., 2019).

\section{Asplenium obovatum Viv. subsp. obovatum}

A. obovatum Viv. subsp. obovatum var. protobillotii Demiriz, Viane \& Reichst. - Asplenio obovato

H ros - Steno-Medit.-Macarones.

Shady cliffs, walls and consolidated debris, from 0 to $800 \mathrm{~m}$ a.s.l. - R - LC.

LIG, TOS, LAZ, CAM, CAL, SAR, SIC.

PA: Ustica (Lojacono PAL!), Castelbuono ai Monticelli (Minà PAL!); TP: Isola di Pantelleria (PAL!), Pantelleria a Fossa del Russo, M. grande, Bugeber, Khaggiar; ME: Isole Eolie (Alicudi, Filicudi, Salina, Lipari, Panarea, Vulcano, Stromboli) (Lojacono PAL!), Capo d'Orlando (PAL!), Finale, Milazzo, Tindari ai Laghetti di Marinello, Messina (PAL!), M. Antennammare, Salice, Mandanaci, Peloritani a M. Scuderi presso punt.le Puzzu (Raimondo PAL!), presso Camaro (PAL!), Taormina, M. Ziretto, M. Lapa; CT: Catania (PAL!), Mascali, S. Tecla di Acireale, Acireale (Nicotra PAL!), Timpa di Acireale, Fiume Alcantara, V.ne Ulli, Etna (PAL!), Etna colata del 1381, Catania Margi (PAL!), Catania Margi presso Vasadonna (PAL!);

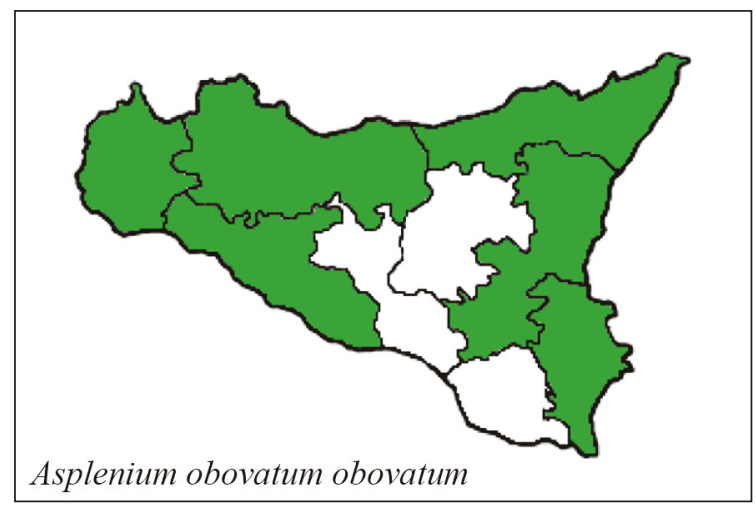

AG: Isola di Linosa presso Mannarazza; SR: Riserva Naturale Orientata "Pantalica, Valle dell'Anapo e Torrente Cava Grande " (Gussone, 1845; Lojacono, 1909; Gianguzzi, 1999, 2003; Marchetti, 2004; Raimondo et al., 2004; Minissale et al., 2007; Giardina et al., 2007; Legambiente, 2009; Giardina, 2010; Licandro et al., 2011; Sciandrello et al., 2013, 2014; Domina et al., 2019).

Asplenium obovatum Viv. subsp. lanceolatum (Fiori) P. Silva

Asplenium billotii F.W. Schultz; A. lanceolatum Huds.; A. fontanum Bernh. var. lanceolatum Fiori; A. obovatum subsp. billotii (F.W. Schultz) O. Bolòs, Vigo, Masalles et Ninot. - Asplenio lanceolato, Asplenio di Billlot

H ros - SW-Europ.

Walls, cliffs and consolidated debris, from 0 to $1100 \mathrm{~m}$ a.s.l. - R - CR.

VEN, VDA, LIG, TOS, LAZ, CAM?, CAL?, SAR, SIC.

PA: Manche di Caltavuturo (PAL!), Isnello (PAL!); TP: Pantelleria (Sommier FI!); ME: Messinese, Portella dell'Arena (PAL!) (Gianguzzi,

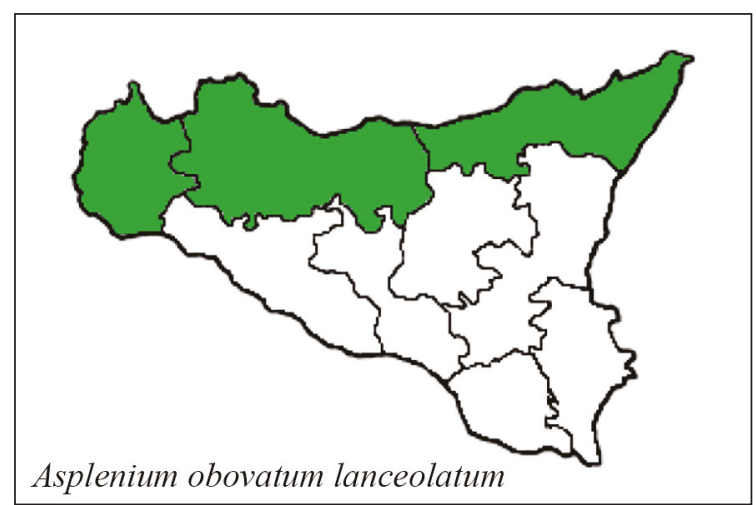




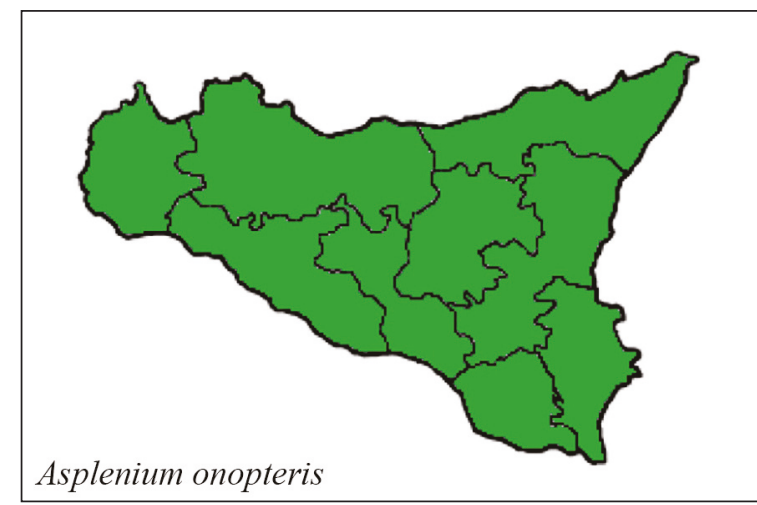

1999; Raimondo \& al., 1992; Gianguzzi, 2003; Marchetti, 2004; Giardina et al., 2007; Troia et al., 2012b; Domina et al., 2019).

\section{Asplenium onopteris L.}

Asplenio maggiore

H ros - Steno-Medit.-Macarones.

Cliffs, walls, cracks in the rock, holm oak woods, from 0 to $1500 \mathrm{~m}$ a.s.1. - CC - LC.

FVG, VEN, TAA, PIE, LIG, EMR, TOS, MAR, UMB, LAZ, ABR, MOL, CAM, PUG, BAS, CAL, AR, SIC.

\section{PA, TP, CT, ME, EN, AG, CL, RG, SR.}

Note. Individuals of a humid and shady environment are particularly large and slender. In dry and sunny places there are more compact morphologies that are similar to those of $A$. adiantumnigrum subsp. adiantum-nigrum, with which, on a general level, there is often confusion (Marchetti, 2004). For this reason A. adiantumnigrum subsp. adiantum-nigrum is absent in Sicily.

Asplenium petrarchae (Guérin) DC. subsp. petrarchae

Polypodium petrarchae Guérin; Asplenium glandulosum Loisel. - Asplenio ghiandoloso, Asplenio di Petrarca

H ros - Steno.-Medit-Occid.

Limestone cliffs, walls, rocky crevices, from 0 to $1000 \mathrm{~m}$ a.s.l. - $\mathrm{R}$ - EN.

LIG, TOS, LAZ, CAM, BAS, CAL, SAR, SIC.

PA: Palermo e dintorni (Marinetti; Todaro

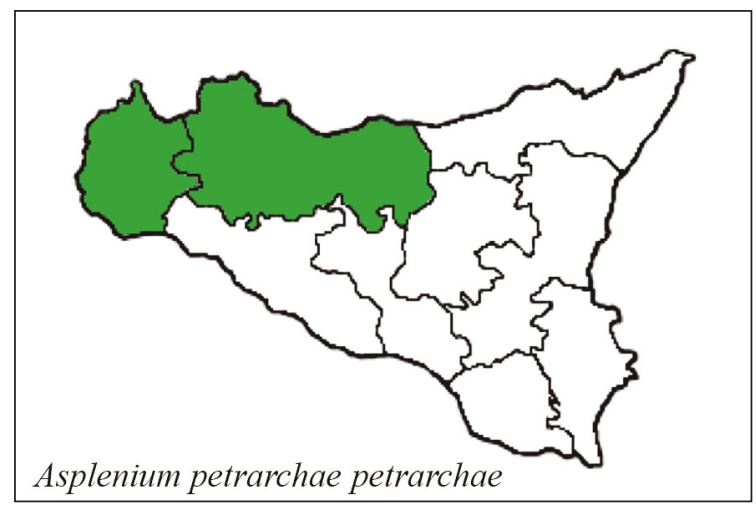

PAL!), M. Pellegrino (PAL!), M. Gallo (Lojacono; Tineo PAL!), M. Gallo lato Tommaso Natale, vetta di M. Cuccio, Catena della Rocca, S. Martino delle Scale, Capo Zafferano, Madonie presso Mandria Castiglia (Porcari PAL!); TP: Riserva dello Zingaro a Tonnarella dell'Uzzo, M. Cofano (FI!), presso Trapani (Gussone, 1845; Lojacono, 1909; Raimondo et al., 1992; Raimondo et al., 1993; Marchetti, 2004; Raimondo et al., 2004; Giardina et al., 2007; Marchetti et al., 2010; Domina et al., 2019).

Asplenium ruta-muraria L. subsp. ruta-muraria Asplenio Ruta di muro

H ros - Circumbor.-Temp.

Limestone cliffs, walls, rocky crevices, from 0 to $2800 \mathrm{~m}$ a.s.l. - RR - VU.

FVG, VEN, TAA, LOM, PIE, VDA, LIG, EMR, TOS, MAR, UMB, LAZ, ABR, MOL, CAM, PUG, BAS, CAL, SAR, SIC.

PA: M. Gallo (Tineo PAL!), Rocca Busambra, M. Quacella, Madonie (Tineo PAL!), Sala Madaggio, Pietrafucile, Serra dei Daini, rupi di Isnello, Madonie al Balzo Canna (PAL!), M. San Calogero

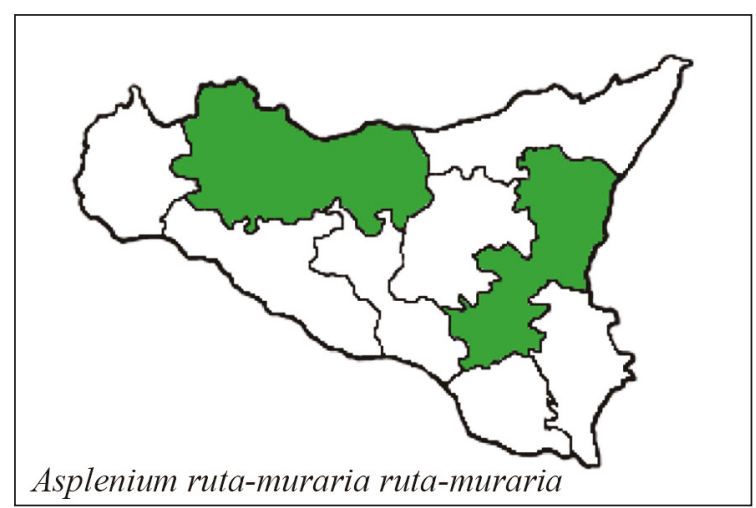




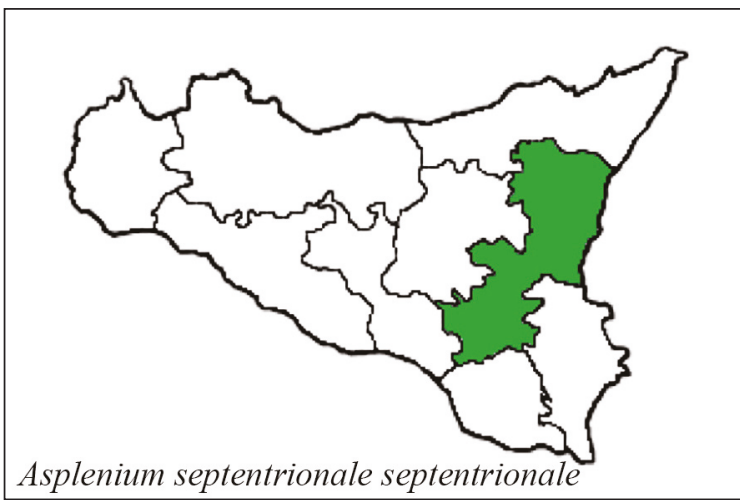

(Madonie) e Rocca di Mele; CT: M. Ciraulo, Etna (Gussone, 1845; Lojacono, 1909; Turrisi, 2004; Giardina et al., 2007).

Asplenium septentrionale (L.) Hoffm. subsp. septentrionale

Acrostichum septentrionale L. - Asplenio settentrionale

$\mathrm{H}$ ros - Circumbor.

Rocky crevices, walls, acid substrates, from 0 to $2800 \mathrm{~m}$ a.s.l. - R - VU.

FVG, VEN, TAA, LOM, PIE, VDA, LIG, EMR, TOS, MAR?, LAZ, BAS, CAL, SAR, SIC.

CT: Catania sull'Etna (G. Certa et al. PAL!), Etna orientale, Etna presso bocca subterminale di NE del 1911 (Certa et al. PAL!), Etna Croce di Pietra (PAL!), M. Pomiciaro, Balzo del Trifoglietto, Grotta del Gelo, M. Silvestri, Grotta Intraleo, a sud di M. Testa, Serra la Neve, a sud di M. Salto del Cane, Sciara del Follone, Etna presso C.da Previtera a Zafferana Etnea (Certa et al. PAL!), sopra Bosco di Nicolosi, vicino al Grande Albergo dell'Etna Gussone, 1845 (Lojacono, 1909; Raimondo et al., 1992; Marchetti, 2004; Marchetti, 2004; Raimondo et al., 2004; Giardina et al., 2007; Giardina, 2010; Domina et al., 2019).

Asplenium trichomanes L. subsp. inexpectans Lovis - Asplenium microphyllum Tineo; A. trichomanes-ramosum L. (erroneous name in Giardina et al. 2007) - Asplenio tricomane inaspettato

H ros - Europ.

Rocky walls, crevices, walls, from 200 to 350 m a.s.l. - R - VU.

FVG, TAA, LIG, SAR, SIC.

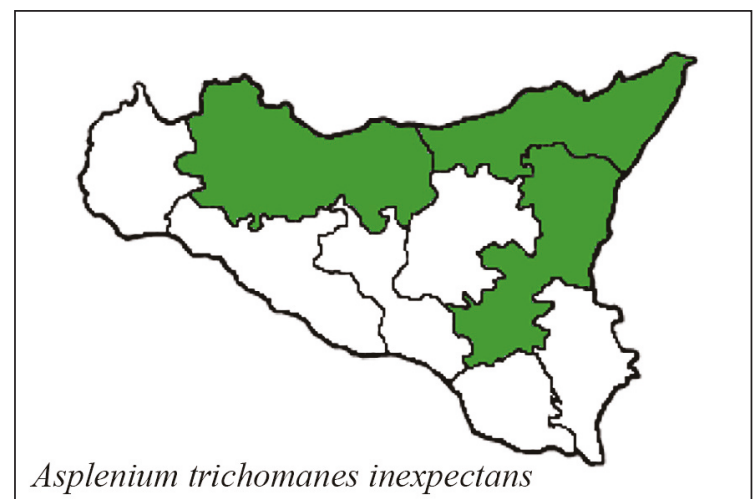

PA: Presso Palermo (Todaro PAL!), Rupi calcaree presso Palermo (Todaro PAL!), Strasatto, M. Gallo (Tineo PAL!), M. Pellegrino (PAL!), S. Martino (PAL!), Ficuzza (Lojacono PAL!), Busambra (PAL!), Bosco e rocche di Carini (PAL!), rupi delle Scale di Mezzagno (Tineo PAL!), M. Pizzuta, Monte Carcaci (Raimondo et al. PAL!), Bosco Guscigliera, Fiume Sosio, Chiusa Sclafani (Certa PAL!), Madonie (PAL!), Mandra del Conte a Piano Zucchi, Monticelli (PAL!); ME: Vulcano - Vallonaccio (Lojacono PAL!), Mirto (Todaro PAL!); CT: Catania (PAL!), V.ne Reale (PAL!), Etna (Certa et al. PAL!), Nicolosi (PAL!), Vaccarizzo (PAL!) (Gussone, 1845; Lojacono, 1909; Raimondo et al., 1993; Giardina et al., 2007; Marchetti et al., 2010).

Note. In Sicily and Sardinia there is an extreme form called Asplenium microphyllum Tineo: mainly thermophilic and non-silicicolous, diploid. It has shorter fronds, with elongated pinnules and crenatelobulated margins. The smaller spores have a clear perisporium with little raised ridges. Due to its general morphology it falls within the Asplenium trichomanes group, but further taxonomic studies are needed.

Asplenium trichomanes L. subsp. pachyrachis (Christ) Lovis \& Reichst. -

Asplenio tricomane dal grosso rachide

$\mathrm{H}$ ros - C/S-Europ.

Rocky walls, crevices, walls, from 280 to 1600 m a.s.l. - RR - DD.

FVG?, VEN, TAA, LOM, LIG, MAR, LAZ, ABR?, MOL?, SAR?, SIC?

PA?: Madonie a Piano Zucchi, Portella Chiusa. 


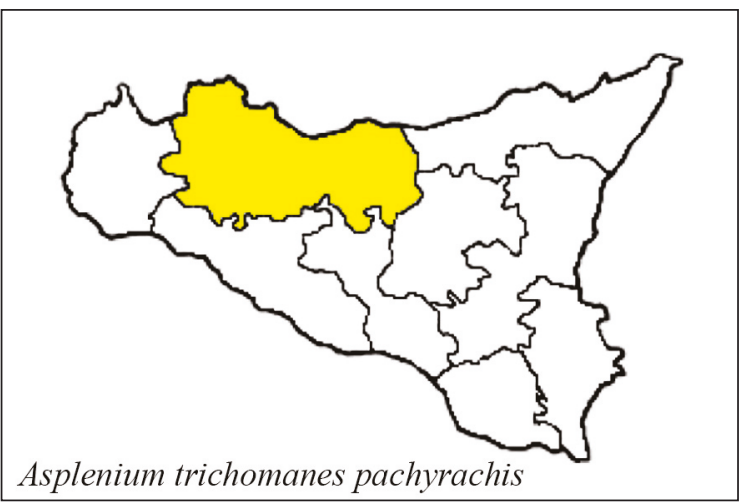

Not found recently, its presence in Sicily is uncertain (Marchetti, 2004; Giardina et al., 2007).

Note. Morphologically very variable plant whose real distribution is not yet well known (Marchetti, 2004; Pignatti et al., 2017/19). According to Pignatti et al. (2017/19), the presence of this plant in Sicily should be eliminated because the report for the Madonie is based on a diploid finding, following a check of the size of the spores.

Asplenium trichomanes L. subsp. quadrivalens D.E. Mey.

Asplenio tricomane quadrivalente

H ros - Cosmop.-Temp.

Rocky walls, crevices, walls, debris, earthy slopes, from 200 to $2000 \mathrm{~m}$ a.s.1. - CC - LC.

FVG, VEN, TAA, LOM, PIE, VDA, LIG, EMR, TOS, MAR, UMB, LAZ, ABR, MOL, CAM, PUG, BAS, CAL, SAR, SIC.

PA: Isola di Ustica, Palermo (PAL!), M. Catalfano, Capo Zafferano, Riserva M. Cane, Monte S. Calogero a Termini Imerese (!) (Certa \& D’Agostino

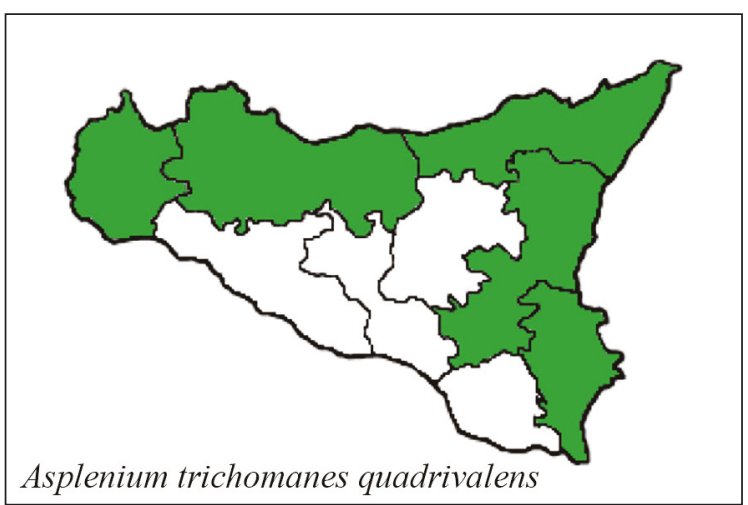

PAL!), Rocca Busambra (PAL!), M. Carcaci, Madonie, Vicaretto ad Acqua Fetente, Monticelli, Val.ne Cartiera; TP: Isola di Marettimo (PAL!), Isola di Pantelleria, M. Cofano; ME: Isola di Vulcano, Valdemone (Citarda PAL!), Messina, M. Scuderi, V.ne Mandraza (S. Lucia del Mela, M. Peloritani); CT: Catania, Nicolosi, S. Michele, Etna, M. Ciraulo; SR: Riserva Naturale Orientata 'Pantalica, Valle dell'Anapo e Torrente Cava Grande", Monti Climiti (Lojacono, 1909; Gramuglio et al., 1978; Raimondo et al., 2004; Turrisi, 2004; Gianguzzi et al., 2005, 2006; Giardina et al., 2007; Minissale et al., 2007; Zimmitti et al., 2007; Gristina \& Marcenò, 2008; Troia et al., 2012b; Marino et al., 2014; Domina et al., 2019).

\section{Genus Ceterach Willd.}

Ceterach officinarum Willd. subsp. officinarum Asplenium ceterach L. subsp. ceterach - Calcedraccia comune

H ros - Eurasiat.-Temp.

Cliffs, walls, fissures and consolidated debris, from 0 to $2000 \mathrm{~m}$ a.s.1. - CC - LC.

FVG, VEN, TAA, LOM, PIE, VDA, LIG, EMR, TOS, MAR, UMB, LAZ, ABR, MOL, CAM, PUG, BAS, CAL, SAR, SIC.

PA, TP, CT, ME, EN, AG, CL, RG, SR.

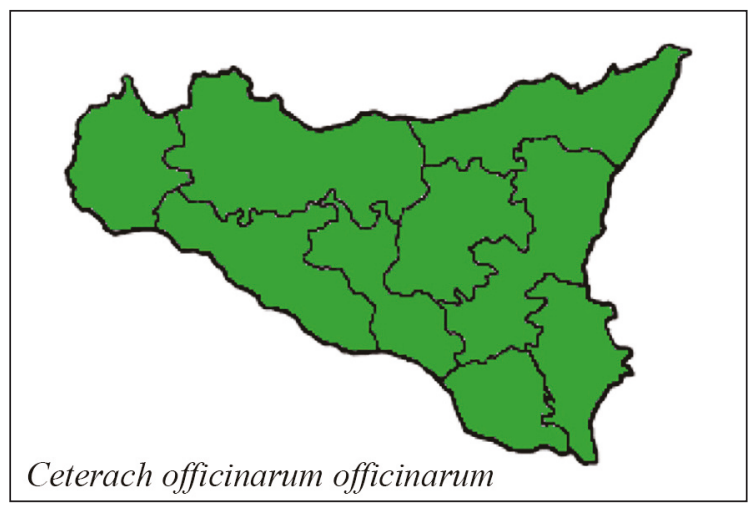

Ceterach officinarum Willd. subsp. bivalens D.E. Mey

Asplenium ceterach L. subsp. bivalens [D.E. Mey] Greuter \& Burdet; A. javorkeanum Vida. - Calcedraccia comune bivalente

H ros - Euri-Medit.-Mont. 


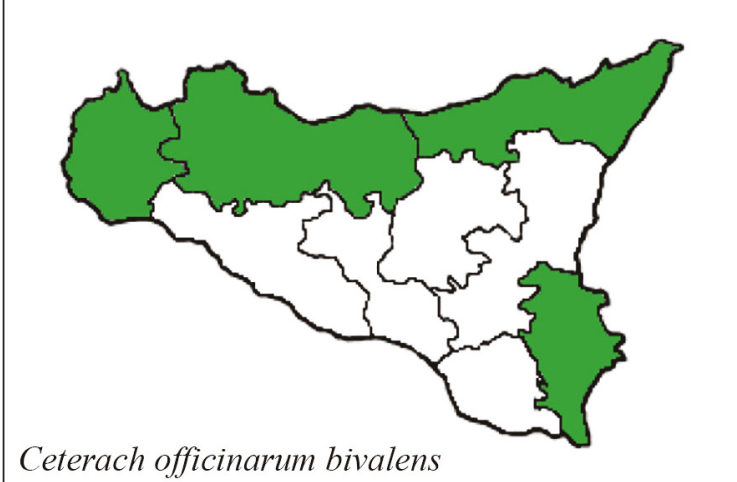

Cliffs, walls, fissures and consolidated debris, from 0 to $1600 \mathrm{~m}$ a.s.l. - RR - LC.

FVG, VEN, EMR, TOS, MAR, UMB, LAZ, ABR, MOL, CAM, PUG, BAS, CAL, SAR, SIC.

PA: Rupi calcaree di Palermo (PAL!), rupi di M. Gallo (PAL!), Ficuzza, Rocca Busambra, Sorgente Cucco, M. San Calogero (Termini Imerese) (G. Marcellino \& L. D’Agostino PAL!), Madonie (Bonafede PAL!), Dirupi di Isnello (PAL!), Pizzo Carbonara (Certa et al. PAL!), Piano Zucchi, Mandra del Conte; TP: M. San Giuliano vicino Erice (Raimondo PAL!); ME: messinese; SR: Riserva Naturale Orientata "Pantalica, Valle dell'Anapo e Torrente Cava Grande" (Pignatti, 1982; Raimondo et al., 1992; Marchetti, 2004; Giardina et al., 2007; Minissale et al., 2007; Marchetti et al., 2010).

Ceterach officinarum Willd. subsp. cyprium (Viane \& Van den heede) Marchetti

Asplenium ceterach L. subsp. mediterraneum Pinter.; A. crypium Viane \& Van den heede - Calcedraccia comune di Cipro

H ros - Steno-Medit.

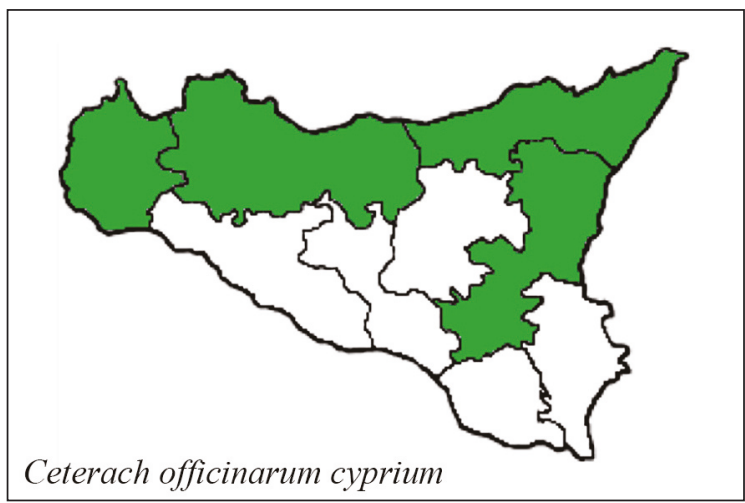

Cliffs, walls, fissures and consolidated debris, from 0 to $250 \mathrm{~m}$ a.s.l. - R - LC.

PUG, SAR, SIC

PA: Rupi calcaree di Palermo (PAL!), M. Pellegrino (PAL!), M. Gallo, sopra San Ciro (PAL!), Monreale (Roccobono PAL!), Rocca Busambra (Raimondo PAL!), M. San Calogero (Caccamo) (G. Certa PAL!); TP: Pantelleria, Erice; ME: Taormina a Lido Spisone, Mazzarò; CT: Zafferana Etnea al Rifugio Sapienza, Acireale (Marchetti et al., 2010).

\section{Genus Phyllitis Hill}

Phyllitis sagittata (DC.) Guinea \& Heywood Scolopendrium sagittatum DC.; Asplenium sagittatum (DC) Bange; Asplenium hemionitis L.; Phyllitis hemionitis (Sw.) Kuntze; Scolopendrium hemionitis (Sw.) Lag, García \& Clemente - Scolopendria emionitide, Asplenio sagittato

H ros - Steno-Medit.

Cave, rock fissures, wells, walls, humid and shady environments, from 0 to $600 \mathrm{~m}$ a.s.l. - RR VU.

TOS, LAZ, CAM, PUG?, CAL, SAR, SIC.

PA: Santa Maria di Gesù, San Martino, grotta di M. Pizzuta (Marcenò PAL!), Madonie, M. Sicani, Partinico presso il fiume Nocella (Gianguzzi PAL!), C.da Chiusa di Landro (Carini) (Scomparsa a M. Pellegrino, M. Gallo e a Ficuzza); TP: Marettimo a Pizzo Falcone e rupi di Punta Lisandro, M. Cofano, M. San Giuliano (Erice), San Vito Lo Capo (Cala Firriato), M. Scardina presso Scopello, Riserva dello Zingaro alla Tonnarella dell'Uzzo; CT: Grotta della Cusaria (Caltagirone) (Scomparsa a San Michele alla Grotta Minnelli; RG: Spaccaforno (Ispica); SR: Riserva Naturale Orientata

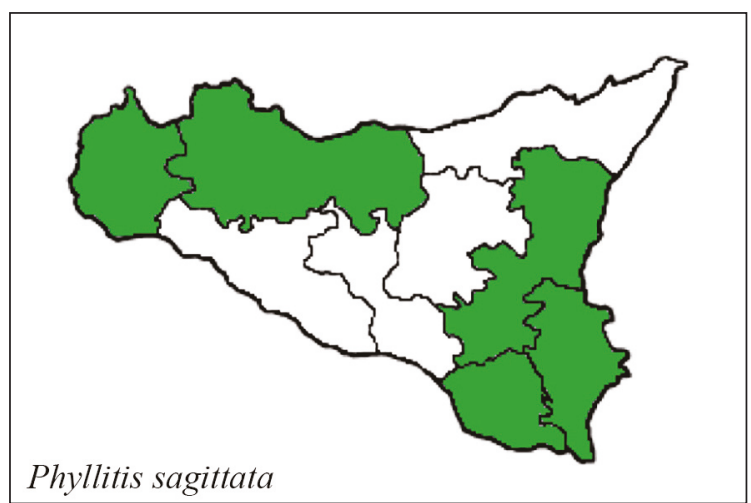


Pantalica, Valle dell'Anapo e Torrente Cava Grande (Scomparsa nel sito dell'anfiteatro di Siracusa e a Capo Passero) (Gussone, 1845; Lojacono, 1909; Mazzola \& Ottonello, 1979; Ottonello \& Catanzaro, 1985; Raimondo et al., 1992; Raimondo et al., 1993; Marchetti, 2004; Raimondo et al., 2004; Gianguzzi et al., 2005, 2006; Giardina et al., 2007; Minissale et al., 2007; Troia et al., 1012b; Domina et al., 2019).

Note. Some stations mentioned above require verification (Giardina et al., 2007).

Phyllitis scolopendrium (L.) Newman subsp. scolopendrium

Asplenium scolopendrium L. subsp. scolopendrium; Scolopendrium officinale Sw.; S. vulgare Sm. Scolopendria comune, Lingua cervina, Asplenio scolopendrio

H ros - Circumbor.-Temp.

Shady woods, cave, rock fissures, wells, walls, humid and shady environments, from 0 to $1300 \mathrm{~m}$ a.s.l. - RR - NT.

FVG, VEN, TAA, LOM, PIE, VDA?, LIG, EMR, TOS, MAR, UMB, LAZ, ABR, MOL, CAM, PUG, BAS, CAL, SAR, SIC.

PA: M. Pizzuta presso Grotta del Garrone! (Raimondo PAL!), San Martino (Roccobono PAL!), Boccadifalco (Parlatore FI!), Ficuzza (Todaro PAL!), Ficuzza al V.ne Arcera, Ficuzza al V.ne Cerasa, Ficuzza alla Grotta del Romito (Lojacono PAL!), Busambra (Raimondo PAL!), Madonie in Contrada Giumenti, Madonie al Passo della botte (PAL!), Castelbuono (Minà FI!, PAL!), Madonie V.ne Canna (Raimondo et al. PAL!); TP: Isola di Favignana, Isola di Pantelleria (extinct in Mrettimo), Scopello; ME: Messina, Parco dei Nebrodi,

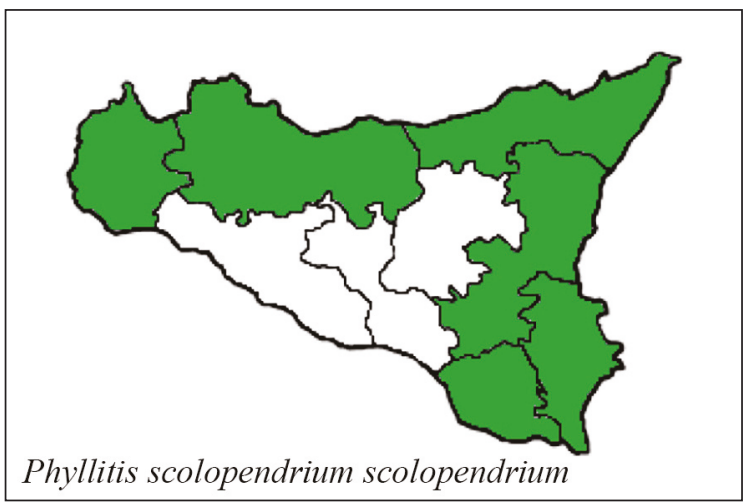

Castroreale, Novara, S. Agata di Militello, Valle dell'Ossena, Salto dell'Acero, S. Lucia del Mela al V.ne Mandrazza (M. Peloritani), Nebrodi al bosco della Tassita, Peloritani a Pizzo Toscano, V.ne Soldato, V.ne Santissima (Fiumedinisi); CT: Catania (CAT!), territorio circostante l'Etna, Caltagirone, alla grotta di Carperia (NAP!), Milo, Zafferana, Bronte, Nicolosi, Mascalucia, Paternò; RG: Comune di Ragusa, Ispica, Cava Misericordia; SR: Siracusa (Minà PAL!), Riserva Naturale Orientata Pantalica, Valle dell'Anapo e Torrente Cava Grande, Monti Climiti; Acquedotto Galermi (PAL!), Palazzolo Acreide a cava Bauli; AG: extinct in Lampedusa: Gussone, 1845; Lojacono, 1909; Gramuglio et al., 1978; Mazzola \& Ottonello, 1979; Gianguzzi, 2003; Gianguzzi \& La Mantia, 2004; Raimondo et al., 2004; Schicchi, 2004; Gianguzzi et al., 2006; Giardina et al., 2007; Minissale et al., 2007; Zimmitti et al., 2007; Licitra \& Napoli, 2011; Troia et al., 2012b; Barone, 2016; Occhipinti, 2018; Domina et al., 2019.

\section{BLECHNACEAE Newman}

\section{Genus Blechnum L.}

Blechnum spicant (L.) Roth

Osmunda spicant L.; Blechnum boreale Sw.; Struthiopteris spicant (L.) Weiss. - Lonchite minore, Blechnum spigato

$\mathrm{H}$ ros - Euro-Americ.-Anfi-Atlant.

Woods, wet slopes, shores of streams, on acid substrate, from 50 to $1900 \mathrm{~m}$ a.s.l. - RR - LC.

FVG, VEN, TAA, LOM, PIE, VDA?, LIG, EMR, TOS, MAR, LAZ, ABR, MOL, CAM, CAL, SAR, SIC.

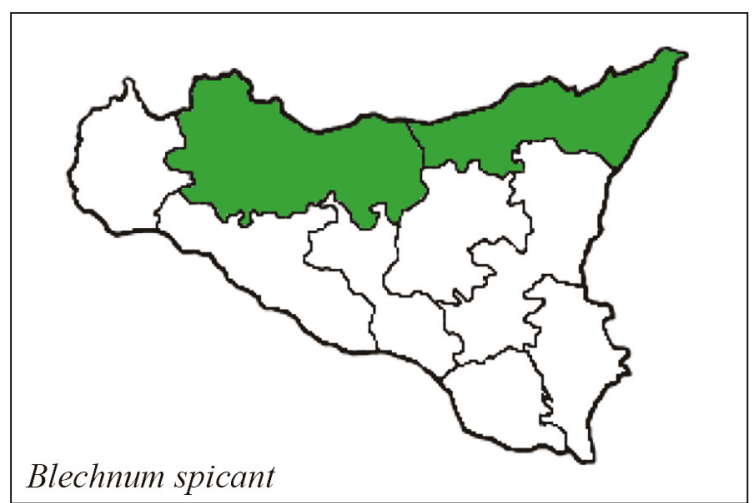


PA: Madonie (Ross PAL!), Monticelli (PAL!), Margi di Badia, Madonna dell'Alto (PAL!), V.ne sotto Rocca di Mele (Ross PAL!), Lercara Friddi, Castelbuono V.ne del Canaliccio (Ross; Lojacono PAL!), Castelbuono ai Russelli, Piano Pomo, Madonie Margio dello Scorzone presso Piano Catarineci (Raimondo PAL!), Piano Pomieri, V.ne Ortaggi; ME: Peloritani, Torrente S. Pietro, M. Scuderi (PAL!), Mandanici dopo Portella di Cardali, Bafia, Antennammare, Saponara, Galati di Tortorici, Tortorici all'Acquasanta, Fiumedinisi (Gussone, 1845; Lojacono, 1909; Giardina et al., 2007; Domina et al., 2019).

Genus Woodwardia Sm.

Woodwardia radicans (L.) Sm. Blechnum radicans L. - Felce bulbifera

H ros/NP - Steno-Medit.-Macarones.

Humid walls, caves and deep valley with high availability of water and humidity and poor lighting, from 0 to $700 \mathrm{~m}$ a.s.1. - RR - CR.

CAM, CAL, SIC.

ME: M. Peloritani al V.ne Lacino (ex V.ne dell'Acero) presso S. Lucia del Mela, V.ne Ferrà (S. Lucia del Mela), V.ne Mandrazza (Gianguzzi PAL!), Rocche Jero (Rometta), Puntale Saitta-Tracanali (Rometta), Serro Ginestri-Tracanali (Rometta), S. Pietro-Tracanali (Rometta), Messina alle acque della Valle del Niceto, M. Girasa, Gualtieri-Sicaminò, V.ne Imeli (bacino del Niceto); CT: Mascali nel V.ne Gulli al Muscarello, Etna al Bosco di Milo (Raimondo PAL!), Milo al V.ne Ulli (PAL!), Torrente Fontanelle (Raimondo PAL!) (Gussone, 1845; Lojacono, 1909; Gramuglio et al., 1978; Picone et al., 2003; Raimondo et al., 2004;

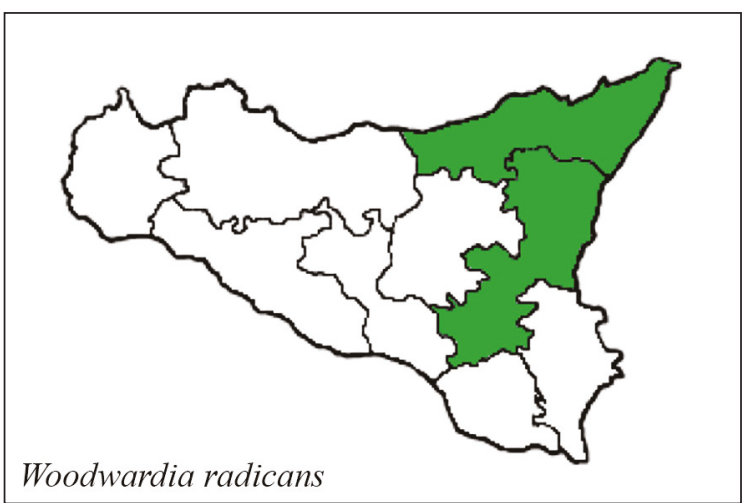

Giardina et al., 2007; Pisani, 2007; Domina et al., 2019).

Note. Relict species of the Tertiary (about 70 million years ago), testimony of the sub-tropical climate of that period. Due to the climatic changes that established the Mediterranean climate, its range has been considerably reduced, finding refuge in the humid and shady valleys, becoming a very rare species and threatened by anthropogenic pressure. Since it is one of the few examples of that ancient flora, it is today to be considered a real "living fossil". For the reasons mentioned above, this fern is protected by international conventions (Berne Convention; Habitat Directive 43/92 CE) and is reported in the Regional Red Lists as a "highly threatened" species.

\section{ATHYRIACEAE Alston}

\section{Genus Athyrium Roth.}

\section{Athyrium filix-femina (L.) Roth}

Polypodium filix-femina L.; Asplenium filix-femina (L.) Bernh. - Felce femmina

H ros - Subcosmop.

Humid woods, streams and waterways, from 0 to $1800(-2000)$ m a.s.l. - NC - LC.

FVG, VEN, TAA, LOM, PIE, VDA, LIG, EMR, TOS, MAR, UMB, LAZ, ABR, MOL, CAM, PUG, BAS, CAL, SAR, SIC.

PA: M. di Palermo C.da Agrifoglio, Madonie (Todaro PAL!), Castelbuono, Coprania (Raimondo et al. PAL!), Castelbuono ne'castagnati, Collesano (PAL!), V.ne Ortaggi, M. Ferro (Raimondo PAL!), Pizzo Catarineci; ME: Isola di Vulcano (PAL!), Isola di Vul-

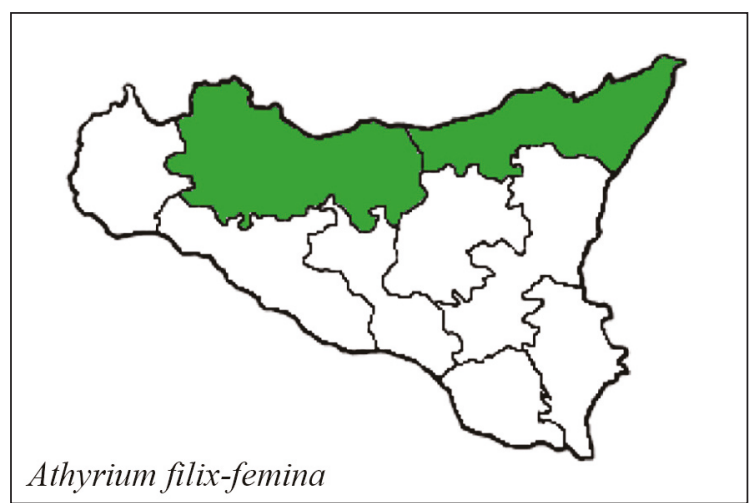


cano alla Grotta di Palisi, Isola di Lipari (Lojacono PAL!), Lipari presso la cima di M. Guardia (Lojacono PAL!), Messina faro superiore (Citarda e Riccobono PAL!), Nebrodi versante nord delle faggete, M. Scuderi (PAL!), Messina nei V.ni del Campo, Messina alla Scala, Mandanici, Antennammare, Fondachelli, Salto dell'Acero, Galati di Tortorici a Cufò, S. Lucia del Mela, Salice, Bosco di Malabotta; Mirto presso C.da S. Andrea (Citarda e Riccobono PAL!), Valle del Mele, Itala (Gussone, 1845; Raimondo et al., 2004; Giardina et al., 2007; Giardina, 2010; Troia et al., 2012b; Domina et al., 2019).

\section{DRYOPTERIDACEAE Herter}

Genus Cyrtomium C. Presl

Cyrtomium falcatum (L. fil.) C. Presil - Polypodium falcatum L. f.; Aspidium falcatum (L. f.) Sw.; Dryopteris falcata (L. f.) Kuntze; Polystichum falcatum (L. f.) Diels - Felce falcata, Cirtomio falcato

G rhiz - E-Asiat. naturalizzata.

Streams, brackish places, cliffs and shady walls, from 0 to $260 \mathrm{~m}$ a.s.l. - RR

TAA, LOM, PIE, LIG, EMR, TOS, CAM, PUG, SIC.

CT: Catania (Pignatti, 2017-19).

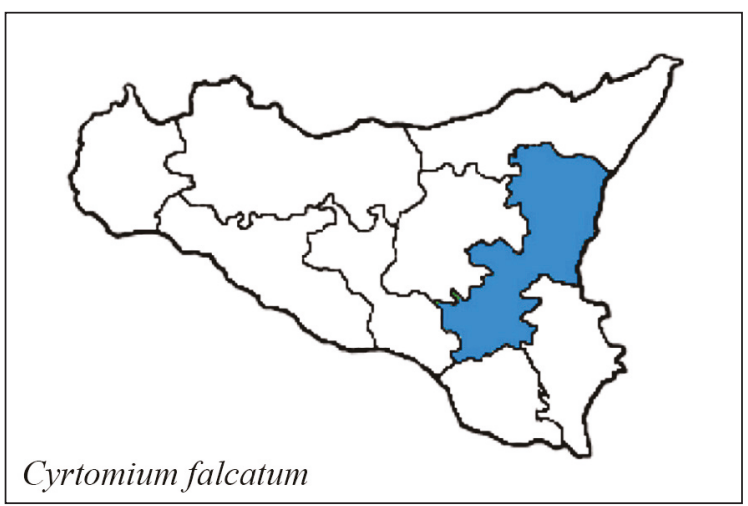

Genus Dryopteris Adans

Dryopteris affinis (Lowe) Fraser-Jenk. subsp. affinis Nephrodium affine Lowe; Dryopteris pseudomas (Woll.) Holub et Pouzar - Felce pelosa

G rhiz - Euri-Medit.-Subatlant.

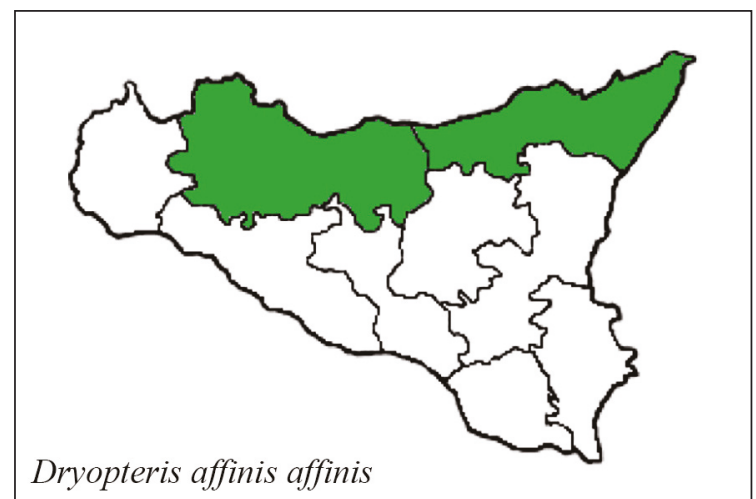

Cliffs, walls, rock fissures, streams, humid and shady woods, from 25 to $1800 \mathrm{~m}$ a.s.1. - R - CR.

LOM, PIE, LIG, TOS, LAZ?, CAM, CAL, SAR, SIC.

PA: Madonie a Vicaretto (Geraci Siculo), Castelbuono ai Canalicchi, Castelbuono a San Guglielmo; ME: Parco dei Nebrodi (Raimondo et al., 1992; Raimondo et al., 2004; Schicchi, 2004; Giardina et al., 2007).

Dryopteris borreri (Newman) Newman ex Oberh. et Tavel

Dryopteris filix-mas var. borreri Newman; D. affinis (Lowe) Fraser-Jenk. subsp. borreri (Newan) Fraser-Jenk.; D. lacunosa S. Jess., Zenner, Chr.Stark \& Bujnoch. - Felce di Borrer

G rhiz - Euri-Medit.-Subatlant.

Cliffs, walls, rock fissures, streams, humid and shady woods, from 25 to $1900 \mathrm{~m}$ a.s.l. - RR - DD.

FVG, VEN, TAA, LOM, PIE, VDA?, LIG, EMR, TOS, MAR, UMB?, LAZ, ABR, CAM, PUG, BAS, CAL, SIC?.

PA?: Madonie: Geraci Siculo a Vicaretto, Ca-

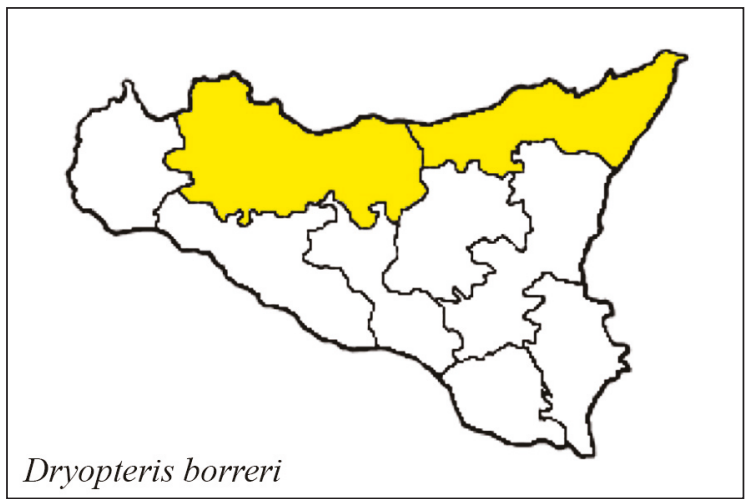


stelbuono al Canalicchio; ME?: (Fiori, 1929; Marchetti, 2004; Giardina et al., 2007). Not found recently, its presence in Sicily is uncertain.

Dryopteris cambrensis (Fraser-Jenk.) Beitel \& W.R. Buck subsp. insubrica (Oberh. \& Tavel ex Fraser-Jenk.) Fraser-Jenk.

Dryopteris affinis (Lowe) Fraser-Jenk. subsp. cambrensis Fraser-Jenk. var. insurbica Oberh. \& Tavel ex Fraser-Jenk. - Felce insubrica

G rhiz - Europ.

Cliffs, walls, rock fissures, streams, humid and shady woods, from 0 to $2900 \mathrm{~m}$ a.s.l. - RR - DD.

FVG, VEN, TAA, LOM, PIE, VDA, LIG, EMR, TOS, MAR, LAZ, CAL?, SAR, SIC?.

Uncertain presence in Sicily.

Note. D. cambrensis subsp. insurbica has never been reported, but according to Fraser-Jenkins (2007) it is also present in Sicily (Pignatti \& al., 2017-19). The actual presence is to be verified (Giardina et al., 2007).

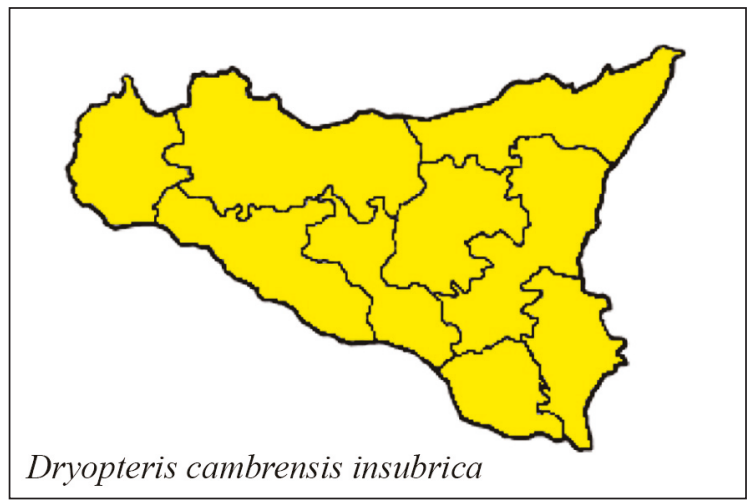

Dryopteris filix-mas (L.) Schott

Polystichum filix-mas (L.) Roth; Nephrodium filixmas (L.) Strempel; Aspidium filix-mas (L.) Sw. Felce maschio

G rhiz - Subcosmop.

Walls, streams, wood, from 0 to $2300 \mathrm{~m}$ a.s.1. $\mathrm{R}$ - LC.

FVG, VEN, TAA, LOM, PIE, VDA, LIG, EMR, TOS, MAR, UMB, LAZ, ABR, MOL, CAM, PUG, BAS, CAL, SAR?, SIC.

PA: Madonie ai Castagneti di Russelli, Castelbuono ai Canalicchi; ME: Nebrodi, S. Lucia del

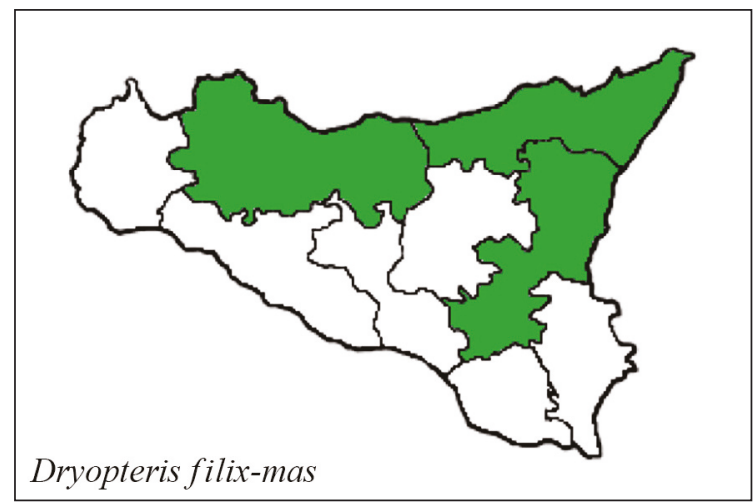

Mela, Caronia, Cannata; CT: Etna, M. Ciraulo (Lojacono, 1909; Turrisi, 2004; Giardina et al., 2007, Sidoti, 2017).

Dryopteris pallida (Bory) C. Chr. Ex Maire \& Petitm. subsp. pallida

Nephrodium pallidum Bory; Dryopteris villarii subsp. pallida (Bory) Heywood - Felce pallida

G rhiz - Medit.-Mont.

Walls, fissures rock, holm oak woods and bushes, from 0 to $2000 \mathrm{~m}$ a.s.1. - C - LC.

LAZ, ABR, MOL, CAM, PUG, BAS, CAL, SAR, SIC.

PA, TP, CT, ME, EN, AG, CL, RG, SR.

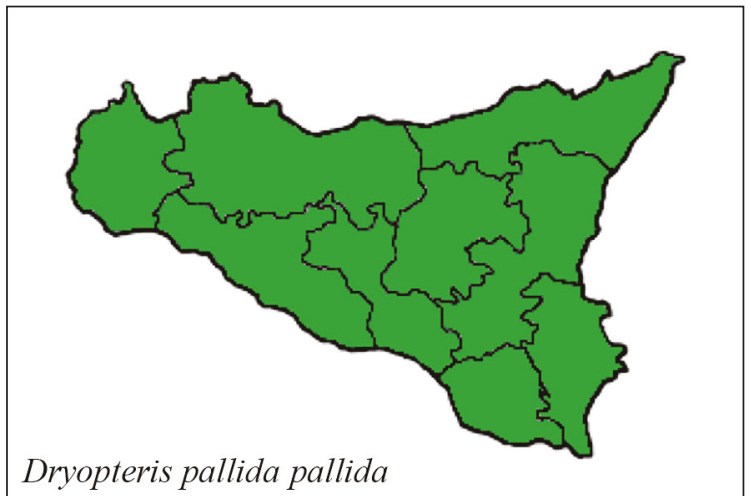

Genus Polystichum Roth.

Polystichum setiferum (Forssk.) Moore ex Woyn. Polypodium setiferum Forssk.; Dryopteris setifera (Forssk.) Woyn.; Polystichum angulare (Kit.) C. Presl.; P. aculeatum (L.) Roth. subsp. angulare Presl; Aspidium aculeatum var. hustulatum (Ten.) Zodda - Felce setifera, Polistico a denti setacei

G rhiz/H ros - Euri-Medit.-Subatlant. 


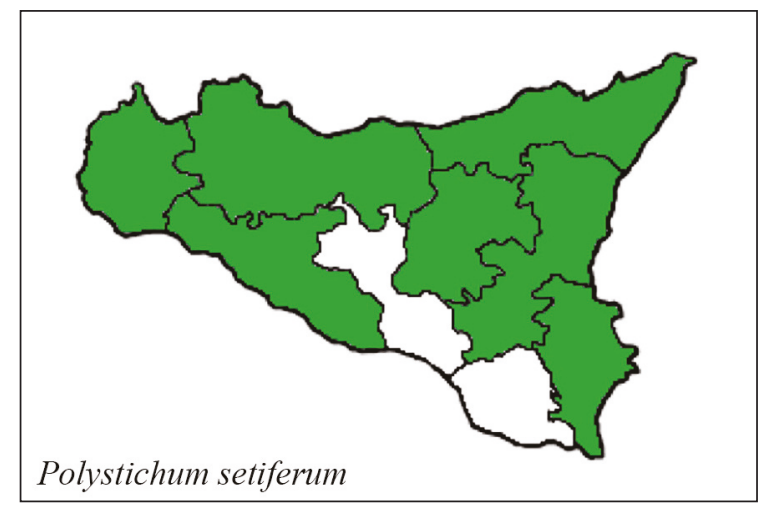

Shady and humid woods, deep valley, streams, from 0 to $2000 \mathrm{~m}$ a.s.l. - NC - LC.

FVG, VEN, TAA, LOM, PIE, LIG, EMR, TOS, MAR, UMB, LAZ, ABR, MOL, CAM, PUG, BAS, CAL, SAR, SIC.

PA: Isola di Ustica, Monti di Palermo a Punte di Cuti, M. Pellegrino, Partinico, Bosco Ficuzza, Rocca Busambra nei macereti sopra la Scala di Ciolino, Madonie, Bosco di Gibilmanna, Polizzi, bosco comunale di Castelbuono, Monticelli (PAL!), Boschi di Cannata, V.ne Scopalacqua, M. Mufara, Pizzo Fao, Contrada Pomieri, Pizzo Canna, Savochella (Portela Mandarini), Portella colla, Sotto il laghetto di Portella Colla (Laghetto di Mandria del Conte), Piano Sempria, Portella di Mele, Cozzo Luminario (Raimondo PAL!), leccete di Palazzo Adriano (MRSN!) Vicaretto ad Acqua Fetente, Monticelli, Val.ne Cartiera, Val.ne Alloro; TP: Alcamo; ME: Isole di Salina, Lipari a M. Sant'Angelo, Vallone Bianco e al Vallone Nord della Costa d'Agosto, Peloritani al V.ne Laneri, V.ne Mandraza a S. Lucia del Mela, Pizzo Toscano al V.ne Soldato, Nebrodi, Pizzo Luminaria, C.da Lannari, C.da Mafauda, Piano Costantino, M. Soro, Messina, Mirto, Mistretta, San Fratello, Antennammare, Bosco di Musolino, Bosco di Malabotta, M. Pomiere; CT: Milo, Randazzo, M. Ilici, Zafferana Etnea, San Tecla, V.ne Ulli, Etna, al Castagno dei Cento Cavalli, M. Spagnolo; EN: M. Sambughetti, Valguarnera, M. Altesina; AG: M. Sicani a M. Genuardo, contrada Rocca Rossa; SR: Valle dell'Anapo (Gussone, 1845; Lojacono, 1909; Gramuglio et al., 1978; Raimondo et al., 1993; Dia et al., 1997; Brullo et al., 1999; Gianguzzi \& La Mantia, 2004; Raimondo et al., 2004; Raimondo et al., 2009; Giardina et al., 2007; Gianguzzi et al., 2011; Troia et al., 1012(b); Marino et al., 2014; Domina et al., 2015;
Gianguzzi et al., 2016; Domina et al., 2019; Cambria \& Tavilla, 2020).

\section{NEPHROLEPIDACEAE Pic. Serm.}

Genus Nephrolepis Schott.

\section{Nephrolepis cordifolia (L.) C. Pres1}

Polypodium cordifolium L.; Aspidium cordifolium (L.) Sw. - Felce pennata; Felce cordifolia

G rhiz - E-Asiat.-Oceanic. Introduced species. Hot and humid places, walls, epiphyte on Phoenix canariensis, from 5 to $275 \mathrm{~m}$ a.s.l. - RR

LIG, TOS, MAR, LAZ, ABR, CAM, CAL, SAR, SIC.

PA: Palermo; ME: Lipari (Di Martino \& Perrone, 1962; Giardina et al., 2007; Cassarà, 2017).

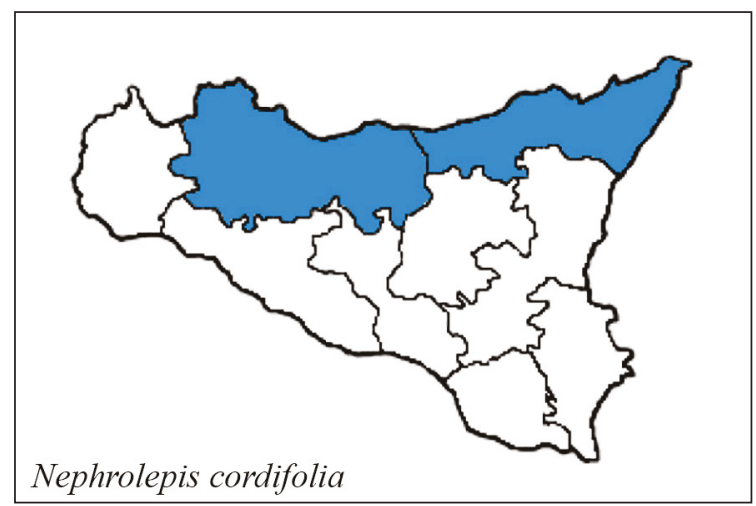

POLYPODIACEAE J. Presl et C. Presl

\section{Genus Polypodium L.}

\section{Polypodium cambricum L.}

Polypodium australe Fée; $P$. australe var. cambricum Willd.; P. serratum (Willd.) Saut.; $P$. vulgare subsp. serrulatum Schinz ex Arcang.; P. cambricum L. subsp. australe (Fée) Greuter \& Burdet; P. cambricum subsp. serrulatum (Schinz ex Arcang.) Pic. Serm. - Polipodio meridionale, Polipodio del Galles

H ros - Euri-Medit.

Cliffs, walls, fissures, woods and anthropic environments, da 0 a $1250 \mathrm{~m}$ a.s.1. - CC - LC.

FVG, VEN, TAA, LOM, PIE, VDA, LIG, EMR, 


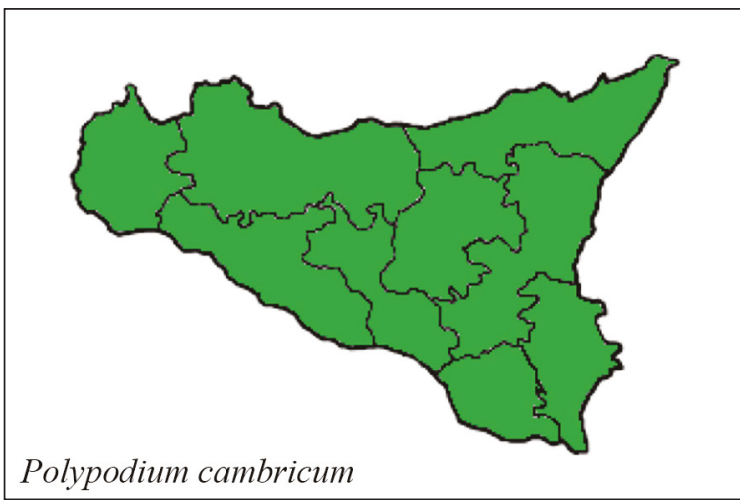

TOS, MAR, UMB, LAZ, ABR, MOL, CAM, PUG, BAS, CAL, SAR, SIC.

PA, TP, CT, ME, EN, AG, CL, RG, SR.

\section{Polypodium interjectum Shivas -}

Polipodio sottile, Polipodio intermedio

H ros - Medit.-Atlant.

Cliffs, walls, fissures, woods, from 0 to $1400 \mathrm{~m}$ a.s.l. - R - VU.

FVG, VEN, TAA, LOM, PIE, VDA, LIG, EMR, TOS, MAR, UMB, LAZ, ABR, MOL, CAM, PUG, BAS, CAL, SAR, SIC.

PA: Monti di Palermo a Punte di Cuti, Madonie, nei pressi di Castelbuono; ME: Nebrodi, C.da Daza presso Frazzano (Gianguzzi PAL!); EN: M. Sambughetti (Nicosia), M. Altesina. (Dia et al., 1997; Raimondo et al., 2004; Giardina et al., 2007; Domina et al., 2019).

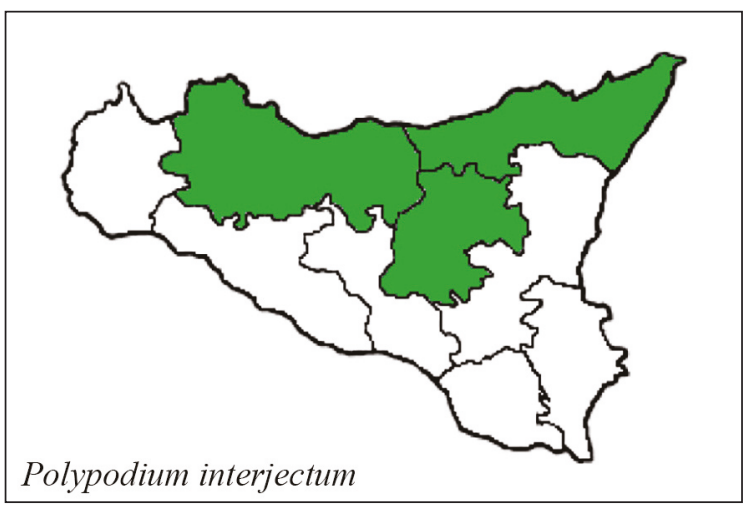

Polypodium vulgare $\mathrm{L}$.

Polipodio comune, Felce dolce, Falsa liquirizia

H ros - Paleotemp.
Cliffs, walls, fissures, woods, from 20 to 2600 m a.s.l. - RR - DD.

FVG, VEN, TAA, LOM, PIE, VDA, LIG, EMR, TOS, MAR, UMB, LAZ, ABR, MOL, CAM, PUG, BAS, CAL?, SAR, SIC?

Note. Uncertain species for Sicily. Historically confused with the other two species of the genus, present in Sicily. In the past, it was mentioned in the literature because the current $P$. cambricum (= $P$. vulgare subsp. cambricum) was also included in this binomial.

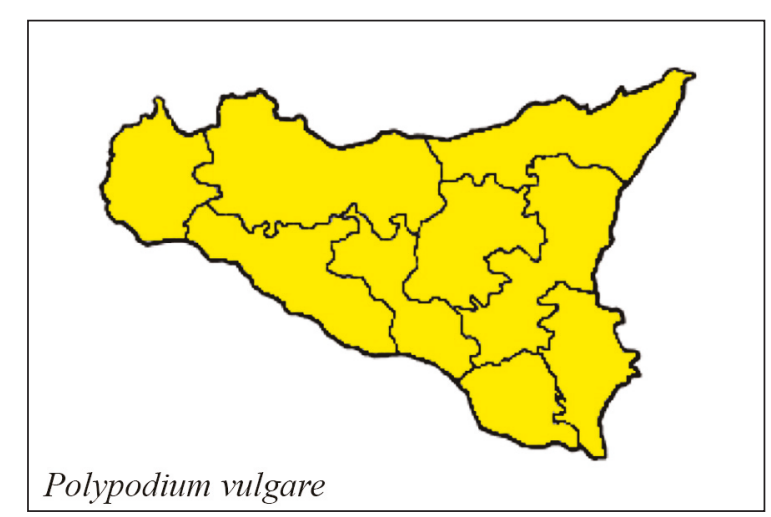

\section{ACKNOWLEDGEMENTS}

I thank Prof. Angelo Troia (University of Palermo, Italy) for the useful suggestions and for the advice in carrying out this work.

\section{REFERENCES}

Albano P.G. (Ed.), 2010. Piano di Gestione del sito "La Gurna e Fiume Fiumefreddo". Ed. 5, Catania, Provincia di Catania.

Aleo M., Bazan G. \& Cordi R., 2004. Le piante vascolari del litorale trapanese: da Capo Lilibeo a Ronciglio. Quaderni di Botanica Ambientale e Applicata, 15: 83-98.

Barone D., 2016. Gruppo Facebook di Flora spontanea siciliana, post_id=1133078330047422, https://www. facebook.com/groups/floraspontaneasiciliana/?post_i $\mathrm{d}=1133078330047422$.

Brullo S., Guarino R., Minissale P., Siracusa G., Spampinato G., 1999. Syntaxonomical analysis of the beech forests from Sicily. Annali di Botanica, 57: 121-132. https://doi.org/10.4462/annbotrm-9052

Cambria S. \& Tavilla G., 2020. Check-list of the vascular flora of the "Bosco di Gibilmanna", a Special Area 
of Conservation (S.A.C.) in northern Sicily (Italy). Biodiversity Journal, 11: 369 -382. https://doi.org/ 10.31396/Biodiv.Jour.2020.11.2.369.382

Cassarà V., 2017. Gruppo Facebook Flora spontanea siciliana. post_id=1394131710608748. https://www. facebook.com/groups/floraspontaneasiciliana/?post_i $\mathrm{d}=1394131710608748$.

Celesti-Grapow L., Alessandrini A., Arrigoni P. V., Banfi E., Bernardo L., Bovio M., Brundu G., Cagiotti M. R., Camarda I., Carli E., Conti F., Fascetti S., Galasso G., Gubellini L., La Valva V., Lucchese F., Marchiori S., Mazzola P., Peccenini S., Poldini L., Pretto F., Prosser F., Siniscalco C., Villani M. C., Viegi L., Wilhalm T. \& Blasi C., 2009. The inventory of the non-native flora of Italy. Plant Biosystems, 143: 386430. https://doi.org/10.1080/11263500902722824

Conti F., Abbate G., Alessandrini A. \& Blasi C. (Eds.), 2005. An Annotated Checklist of the Italian Vascular Flora. Palombi Editori, Roma, 428 pp.

Conti F., Alessandrini A., Bacchetta G., Banfi E., Barberis G., Bartolucci F., Bernardo L., Bonacquisti S., Bouvet D., Bovio M., Brusa G., Del Guacchio E., Foggi B., Frattini S., Galasso G., Gallo L., Gangale C., Gottschlich G., Grünanger P., Gubellini L., Iiriti G., Lucarini D., Marchetti D., Moraldo B., Peruzzi L., Poldini L., Prosser F., Raffaelli M., Santangelo A., Scassellati E., Scortegagna S., Selvi F., Soldano A., Tinti D., Ubaldi D., Uzunov D., Vidali M., 2007. Integrazioni alla checklist della flora vascolare italiana. Natura Vicentina, 10: 5-74.

Dia M.G., Maniscalco M. \& Raimondo F.M., 1997. Caratterizzazione della diversità forestale e briofitica dei Monti di Palermo in rapporto ad indirizzi di gestione naturalistica del territorio. Quaderni di Botanica Ambientale Applicata, 8: 109-125.

Di Martino A. \& Perrone C., 1962. Nuovo contributo alla flora arboricola di Palermo. Lavori dell'Istituto Botanico e del Giardino Coloniale Palermo, 18: 112202.

Domina G., Marino P., Castellano G., Amato F., Cambira S., Cancellieri L., Crisafulli A., Cristaudo A., Faraoni F., Galesi R., Guarino R., Lattanzi E., Lavezzo P., Longo D., Maiorca G., Peccenin S., Perrino E. V., Salerno G., Scolastri A., Soldano A., Stinca A., Wagensommer R.P., Xibilla L. \& Raimondo F.M., 2015. Contributo alla conoscenza floristica dei monti Sicani (Sicilia): resoconto dell'escursione del Gruppo di Floristica (S.B.I.) nel 2012. Informatore Botanico Italiano, 47: 155-177.

Domina G., Peruzzi L. \& Bedini G. (Eds.), 2019. Wikiplantbase \#Sicilia v3.0.

Federico C. \& R., 2015. Guida alla Flora vascolare del complesso collinare Catalfano-Solunto (PA), guida illustrata con 476 foto a colori. Aiello e Provenzano, Bagheria, 281 pp.
Ferro G., Salamone C., Crisci A. \& Finocchiaro G.L., 1992. Piante e aggruppamenti vegetali di notevole interesse naturalistico nell' arcipelago delle Eolie (Sicilia). Quaderni di Botanica Ambientale Applicata, 3: 181-190.

Fiori A., 1929. Nuova Flora Analitica d'Italia, 2. Firenze. Fiori A., 1943: Flora Italica Cryptogama. Pars V: Pteridophyta. Filicinae, Equisetinae, Lycopodinae. Società Botanica Italiana, Firenze.

Fraser-Jenkins C.R., 2007. The species and subspecies in the Dryopteris affinis group. The Fern Gazette, 18: $1-26$.

García Criado M., Väre H., Nieto A., Bento Elias R., Dyer R., Ivanenko Y., Ivanova D., Lansdown R., Molina J., Rouhan G., Rumsey F., Troia A., Vrba J. \& Christenhusz M., 2017. European Red List of Lycopods and Ferns. IUCN, 978-2-8317-1855-2. https://doi.org/10.2305/IUCN.CH.2017.ERL.1.en.

Gianguzzi L., 1999. Vegetazione e bioclimatologia dell'Isola di Pantelleria (Canale di Sicilia). Braun-Blanquetia, 22, Camerino.

Gianguzzi L., 2003. Il paesaggio vegetale dell'isola di Pantelleria. Sicilia Foreste 6, Azienda Foreste Demaniali, Palermo, 192 pp.

Gianguzzi L. \& La Mantia A., 2004. Le serie di vegetazione della Riserva "Bosco Ficuzza, Rocca Busambra, Bosco del Cappelliere e Gorgo del Drago" (Provincia di Palermo) con allegata Carta della Vegetazione (scala 1:20.000). Il Naturalista siciliano, 28: $265-326$.

Gianguzzi L., Caldarella O., Cusimano D. \& Romano S., 2011. Berberido aetnensis-Crataegion laciniatae, new orophilous pre-forestal alliance of the class Rhamno-Prunetea. Phytocoenologia, 41: 183-199. DOI: 10.1127/0340-269X/2011/0041-0492

Gianguzzi L., Cuttonaro P., Cusimano D. \& Romano S., 2016. Contribution to the phytosociological characterization of the forest vegetation of the Sicani Mountains (inland of north-western Sicily). Plant Sociology, 53: 5-43. https://doi.org/10.7338/pls20 16531/02.

Gianguzzi L., La Mantia A., Ottonello D. \& Romano S., 2005. La flora vascolare della riserva naturale Monte Cofano (Sicilia Occidentale). Il Naturalista siciliano, 29: $107-152$.

Gianguzzi L., Scuderi L. \& Pasta S., 2006. La flora vascolare dell'Isola di Marettimo (Arcipelago delle Egadi, Sicilia occidentale): analisi fitogeografica ed aggiornamento. Webbia, 61: 359-402. https://doi. org/10.1080/00837792.2006.10670810

Giordana F., 2013. Forum Acta Plantarum, Topic_id: 55430: Azolla filiculoides Lam. https://floraitaliae.actaplantarum.org/. https://www.floraitaliae.actaplantarum.org/viewtopic.php?t=55430\&p=352498\#p35249 8. 
Giardina G., 2010. Piante rare della Sicilia, Testi e immagini di 500 entità endemiche e rare dell'Isola e dei territori limitrofi. Università degli studi di Palermo Orto Botanico.

Giardina G., Raimondo F. M. \& Spadaro V., 2007. A catalogue of plants growing in Sicily. Bocconea, 20: 5582.

Gramuglio G., Rossitto M., Arena M. \& Villari R., 1978. Nuova stazione di Woodwardia radicans (L.) SM. in Sicilia. Il Naturalista siciliano, 2: 127-134.

Gristina A.S. \& Marcenò C., 2008. Gli indici di bioindicazione di Pignatti-Ellenberg nello studio floristicovegetazionale del promontorio di capo Zafferano (Sicilia nord-occidentale). Il Naturalista siciliano, 32: 61-96.

Gussone G., 1845. Flora Siculae Synopsis. 1-2, Napoli.

Legambiente, 2000. Piano di Gestione "Isole Pelagie" POR 1999.IT.16.1.PO.011/1.11/11.2.9/0347, SIC ITA040001 "Isola di Linosa", SIC ITA040002 "Isole di Lampedusa e Lampione" e ZPS ITA040013 “Arcipelago delle Pelagie. Area marina e terrestre", parte I (Fase Conoscitiva). Regione Siciliana, Assessorato Territorio ed Ambiente, Palermo.

Licandro G., Marino P. \& Raimondo F.M., 2011. Flora e vegetazione della Riserva Naturale Orientata "Laghetti di Marinello" (Sicilia nord-orientale). Informatore Botanico Italiano, 43: 333-351.

Licitra G. \& Napoli M., 2011. Flora spontanea della città di Ragusa. Bollettino Accademia Gioenia Scienze Naturali, 44: 227-278.

Lojacono Pojero M., 1909. Flora Sicula, 3. Palermo.

Luchino F. \& Grioli C., 2016. Gruppo Facebook "Flora spontanea siciliana": post_id=1124618714226717. https://www.facebook.com/groups/floraspontaneasiciliana/?post_id=1124618714226717.

Marcenò C., Colombo P. \& Princiotta R., 1985. Ricerche climatologiche e botaniche sui Monti Sicani (Sicilia centro occidentale): La Flora. Il Naturalista siciliano, 1: 69-133.

Marchetti D., 2004. Le pteridofite d'Italia. Annali del Museo Civico di Rovereto, Sezione: Archeologia Storia Scienze Naturali, 19: 71-231.

Marchetti D. (Ed.), 2008. Note pteridologiche italiche. VI (134-155). Annali del Museo Civico di Rovereto, Sezione: Archeologia Storia Scienze Naturali, 23: 205-226.

Marchetti D., 2009. Note pteridologiche italiche. VII (156-177). Annali del Museo Civico di Rovereto, Sezione: Archeologia Storia Scienze Naturali, 24: 137 152.

Marchetti D., 2010: Note pteridologiche italiche. VIII (178-211). Annali del Museo Civico di Rovereto, Sezione: Archeologia Storia Scienze Naturali, 25: 103 126.

Marchetti D., 2015. Classificazione e distribuzione per regione delle Pteridofite italiane. Annali del Museo Civico di Rovereto, Sezione: Archeologia Storia Scienze Naturali, 31: 137-157.

Marino P., Castiglia G., Bazan G., Domina G. \& Guarino G., 2014. Tertiary relict laurophyll vegetation in the Madonie mountains (Sicily). Acta Botanica Gallica, DOI: $10.1080 / 12538078.2013 .870047$.

Mazzola P. \& Ottonello D., 1979. Distribuzione del genere Phyllitis Hill in Sicilia (Pteridophyta, Aspleniaceae). Il Naturalista siciliano, 3: 103-110.

Médail F., Pavon D., Lo Cascio P. \& Pasta S., 2016. Sulla presenza di Ophioglossum lusitanicum L. (Psilotopsida) nell'isola di Vulcano e aggiunte alla flora vascolare delle isole Eolie (Sicilia Nord-Orientale). Il Naturalista siciliano, 40: 51-66.

Minissale P. \& Sciandrello S., 2017. The wild vascular flora of the Archaeological Park of Neapolis in Syracuse and surrounding areas (Sicily, Italy). Biodiversity Journal, 8: 87-104.

Minissale P., Molina J.A. \& Sciandrello S., 2017. Pilularia minuta Durieu (Marsileaceae) discovered in south-eastern-Sicily: new insights on its ecology, distribution and conservation status. Botany Letters. https://doi.org/10.1080/23818107.2017.1357051.

Minissale P., Sciandrello S. \& Spampinato G., 2005. Analisi della biodiversità vegetale e relativa cartografia della Riserva Naturale Orientata Isola Bella e del territorio circostante (Taormina - ME - Sicilia). Quaderni di Botanica Ambientale Applicata, 16: 175208.

Minissale P., Sciandrello S. \& Spampinato G., 2007. Analisi della biodiversità vegetale e relativa cartografia della Riserva Naturale Orientata "Pantalica, Valle dell'Anapo e Torrente Cava Grande" (Sicilia sudorientale). Quaderni di Botanica Ambientale Applicata, 18: 145-207.

Occhipinti L., 2018: Gruppo Facebook Flora spontanea siciliana: post id=1818082821546966. https://www. facebook.com/groups/floraspontaneasiciliana/?post_i $\mathrm{d}=1818082821546966$.

Oddo E. \& Bellini E., 1994. Some observations on Isoetes durieui Bory (Isoetaceae) in Sicily. Giornale botanico italiano, 128: 359-359. https://doi. org/10.1080/11263509409437182.

Ottonello D. \& Catanzaro F., 1985. Contributo alla flora del trapanese. Il Naturalista siciliano, 9: 89-99.

Picone R.M., Crisafulli A., Zaccone S. \& Damino R., 2003. The flora of Peloritan District (Sicily): contribution to the knowledge of endangered entities distribution. Bocconea, 16: 831-838.

Pignatti S., 1982. Flora d'Italia, 1-3. Edagricole, Bologna.

Pignatti S., Guarino R. \& La Rosa M., 2017-2019. Flora d'Italia, $2^{\circ}$ Edizione. Edagricole, Edizioni Agricole di New Business Media, Bologna. 
Pisani G., 2007. Contributo al censimento di Woodwardia radicans (L.) Sm. in Calabria, Informatore Botanico Italiano, 39: 151-153.

Pteridophyte Phylogeny Group, 2016. A community-derived classification for extant lycophytes and ferns. Journal of Systematics and Evolution, 54: 563-603. https://doi.org/10.1111/jse.12229

Raimondo F. M. \& Spadaro V., 2009. Addenda et emendanda to the "A catalogue of the plants growing in Sicily”. Flora Mediterranea, 19: 303-312.

Raimondo F.M., Bazan G. \& Troia A., 2011. Taxa a rischio nella flora vascolare della Sicilia, Biogeographia, 30: 229-239. https://doi.org/10.21426/ B630110586

Raimondo F.M., Domina G. \& Spadaro V., 2010. Checklist of the vascular flora of Sicily. Quaderni di Botanica Ambientale e Applicata, 21: 189-252.

Raimondo F. M., Gianguzzi L. \& Ilardi V., 1992. Inventario delle specie "a rischio" nella flora vascolare nativa della Sicilia. Quaderni di Botanica Ambientale e Applicata, 3: 65-132.

Raimondo F.M., Gianguzzi L. \& Di Martino C., 1993. La flora vascolare del promontorio di Monte Pellegrino (Palermo). Quaderni di Botanica Ambientale e Applicata, 4: 13-34.

Raimondo F.M., Mazzola P. \& Domina G., 2004. Checklist of the vascular plants collected during iter Mediterraneum III. Bocconea, 17: 65-231.

Raimondo F.M., Schicchi R. \& Bazan G., 2009. Studio fitosociologico dei cerreti con agrifoglio della Sicilia. Il Naturalista siciliano, 33: 373-388.

Raimondo F.M., Venturella G. \& Gianguzzi L., 1990. Lineamenti floristici e vegetazionali del Bacino del Fiume Oreto (Palermo) con annessa carta del paesaggio vegetale (1:50000). Quaderni di Botanica Ambientale e Applicata, 1: 77-91.

Romano S., Ottonello D. \& Marceò C., 1994. Contributo alla floristica siciliana: Nuovi rinvenimenti e ulteriori dati distributivi di alcune entità indigene ed esotiche. Il Naturalista siciliano, 18: 3-14.

Raimondo F. M., Tobia G. \& Gianguzzi L., 2006. Rassegna della flora vascolare dell'Isola di Levanzo (Arcipelago delle Egadi, Canale di Sicilia). Informatore Botanico Italiano, 38: 481-502.

Schicchi R., 2004: Materiali per una carta tematica delle emergenze floristiche e vegetazionali del parco dei Nebrodi. Il Naturalista siciliano, 28: 139-163.

Sciandrello S., D’Agostino S. \& Minissale P., 2013. Vegetation analysis of the Taormina Region in Sicily: a plant landscape characterized by geomorphology variability and both ancient and recent anthropogenic influences. Lazaroa, 34: 151-190. https://doi.org/ 10.5209/rev_LAZA.2013.v34.n1.41434

Sciandrello S., D'Agostino S. \& Minissale P., 2014. The vascular flora of the Taormina Region (Peloritani
Mountains - northeast Sicily). Webbia: Journal of Plant Taxonomy and Geography, 69: 301-324. https://doi.org/10.1080/00837792.2014.966487.

Troia A., 2005. Note corologiche e tassonomiche sul genere Isoëtes L. (Isoëtaceae, Lycophyta) in Sicilia. Informatore Botanico Italiano, 37: 382-383.

Troia A. \& Greuter W., 2015a. A critical conspectus of Italian Lycopodiaceae. Plant Biosystems, 149: 678-694. https://doi.org/10.1080/11263504.2015.1057 263.

Troia A. \& Greuter W., 2015b. Flora Critica D'Italia: Isoetaceae. Fondazione per la Flora Italiana, Firenze, $40 \mathrm{pp}$.

Troia A. \& Greuter W., 2015c. A conspectus of and key to Greek Isoetes (Isoetaceae), based on a reassessment of Haussknecht's gatherings of 1885. Willdenowia, 45: 39-403. https://doi.org/10.3372/wi.45. 45303

Troia A. \& Lansdown R., 2016. The first confirmed population of the globally endangered Pilularia minuta (Marsileaceae) in Sicily. Webbia: Journal of Plant Taxonomy and Geography, 71: 283-286. https://doi. org/10.1080/00837792.2016.1195105.

Troia A. \& Raimondo F.M., 2009. Isoëtes todaroana (Isoëtaceae, Lycopodiophyta), a New Species from Sicily (Italy). American Fern Journal, 99: 239-243. https://doi.org/10.1640/0002-8444-99.4.238

Troia A., Ilardi V. \& Raimondo F.M., 2011. Osservazioni sulla flora pteridologica della Sicilia. Biogeographia, 30: 151-157. https://doi.org/10.21426/B630110607

Troia A., Bazan G. \& Schicchi R., 2012a. Micromorphological approach to the systematics of Mediterranean Isoëtes species (Isoëtaceae, Lycopodiophyta): analysis of the megaspore surface. Grana, 51: 35-43. https: //doi.org/10.1080/00173134.2011.637131.

Troia A., Raimondo F.M. \& Mazzola P., 2012b. Mediterranean Island biogeography: Analysis of fern species distribution in the system of islets around Sicily. Plant Biosystem, 146: 576-585. https://doi.org/10.1080/ 11263504.2011.596168

Troia A., Adragna F., Campisi P., Campo G., Dia M.G., Ilardi V., La Mantia T., La Rosa A., Lo Valvo M., Muscarella C., Pasta S., Pieri V., Scuderi L., Sparacio I., Stoch F. \& Marrone F., 2016. I Pantani di Anguillara (Calatafimi Segesta, Trapani): Dati preliminari sulla biodiversità a supporto della tutela del biotopo. Il Naturalista siciliano, 40: 171-200.

Troia A., Johnson G. \& Taylor C., 2019. A contribution to the phylogeny and biogeography of the genus Isoetes (Isoetaceae, Lycopodiidae) in the Mediterranean region. Phytotaxa, 395: 168-178. https://doi. org/10.11646/phytotaxa.395.3.2.

Troia A., Ilardi V. \& Oddo E., 2020. Monitoring of alien aquatic plants in the inland waters of Sicily (Italy). Webbia: Journal of Plant Taxonomy and Geography, 75: 77-83. https://doi.org/10.36253/jopt-8414. 
Turrisi R.E., 2004. La flora di Monte Ciraulo (Etna) e considerazioni sulla conservazione degli ambienti etnei di bassa quota. Quaderni di Botanica Ambientale Applicata, 15: 61-71.

Zimmitti A., Ronsisvalle F.B.F. \& Ronsisvalle G.A., 2007. Aree d'interesse naturalistico per la rete ecologica dei M.ti Iblei (Sicilia sud-orientale): il territorio dei Monti Climiti (Siracusa). Quaderni di Botanica
Ambientale Applicata, 18: 319-342.

\section{SITOGRAPHY}

https://www.actaplantarum.org

https://www.facebook.com/groups/floraspontaneasiciliana

http://bot.biologia.unipi.it/wpb/sicilia/index.html 